\title{
ENTRANCE TIME FUNCTIONS FOR FLAT SPOT MAPS
}

\author{
V. ANAGNOSTOPOULOU, K. DÍAZ-ORDAZ, O. JENKINSON \& C. RICHARD
}

\begin{abstract}
We classify the dynamics of (orientation-preserving) flat spot maps on the circle, and derive explicit expressions for the function counting the first entrance time into the flat spot. Metric properties of first entrance time functions for the standard flat spot family are analysed in detail, via a computation of conditional expectation with respect to the orbit partition. This facilitates investigation of the median of the entrance time function, proving the surprising result that its first entrance time is constrained to equal either $1,2,4,5$, or 12 , provided the rotation number of the flat spot map does not equal the exceptional values $0, \pm 2 / 7, \pm 3 / 10, \pm 1 / 3, \pm 3 / 8$.
\end{abstract}

\section{INTRODUCTION}

Flat spot maps $T: \mathbb{T} \rightarrow \mathbb{T}$, considered in e.g. [2, 12, 18, 19, 20, 21], are defined to be continuous degree-one maps of the circle $\mathbb{T}$ with the property that some non-empty open interval $F$ (the 'flat spot') is mapped by $T$ onto a single point $v \in \mathbb{T}$, and $\left.T\right|_{\mathbb{T} \backslash \bar{F}}$ is an expanding (i.e. $\inf _{x \in \mathbb{T} \backslash \bar{F}} T^{\prime}(x)>1$ ) $C^{2}$ diffeomorphism onto its image $\mathbb{T} \backslash\{v\}$ (see Figure 1). Because flat spot maps are continuous and weakly order-preserving, they have a rotation number

$$
\varrho(T)=\lim _{n \rightarrow \infty} \frac{\tau^{n}(x)}{n} \quad(\bmod 1),
$$

which is well-defined and independent of the choice of continuous lift $\tau: \mathbb{R} \rightarrow \mathbb{R}$ of $T$ and point $x \in \mathbb{R}$ (see e.g. $[6,11,16]$ ).

For a closed $T$-invariant subset $A \subset \mathbb{T}$, we say $x \in \mathbb{T}$ is absorbed by $A$ if there exists $n=n(x) \in \mathbb{N}$ such that $T^{n}(x) \in A$. Such an $A$ will be called totally absorbing if every $x \in \mathbb{T}$ is absorbed by $A$, and absorbing if all but finitely many $x \in \mathbb{T}$ are absorbed by $A$. The dynamics of flat spot maps can be classified as follows:

Theorem 2.1. ([2, 4, 19, 21]) Let $T: \mathbb{T} \rightarrow \mathbb{T}$ be a flat spot map. If $\varrho(T)$ is irrational then there exists a totally absorbing $T$-invariant Cantor subset of $\mathbb{T} \backslash F$. If $\varrho(T)$ is rational then either (i) a single periodic orbit is totally absorbing, or (ii) there are precisely two periodic orbits, one of which is absorbing and absorbs all points of $\mathbb{T}$ except those points in the other periodic orbit.

The points absorbed by the absorbing invariant set in Theorem 2.1 are precisely those which eventually enter the flat spot $F$ under some iterate of $T$. The time taken to reach $F$ is the main focus of this paper: in Theorem 3.13 we give an explicit description of the first entrance time function $e_{T}$, defined by $e_{T}(x)=\inf \left\{i \geq 0: T^{i}(x) \in F\right\}$ (cf. Figures 2, 3 and 5). Although first entrance time functions (for arbitrary maps $T$ and subsets $F$ of phase space) have been studied widely in the dynamical systems literature (see e.g. [1, 5, $7,8,14,17]$ and Remark 3.24), it is rarely feasible to obtain explicit descriptions of these functions. 

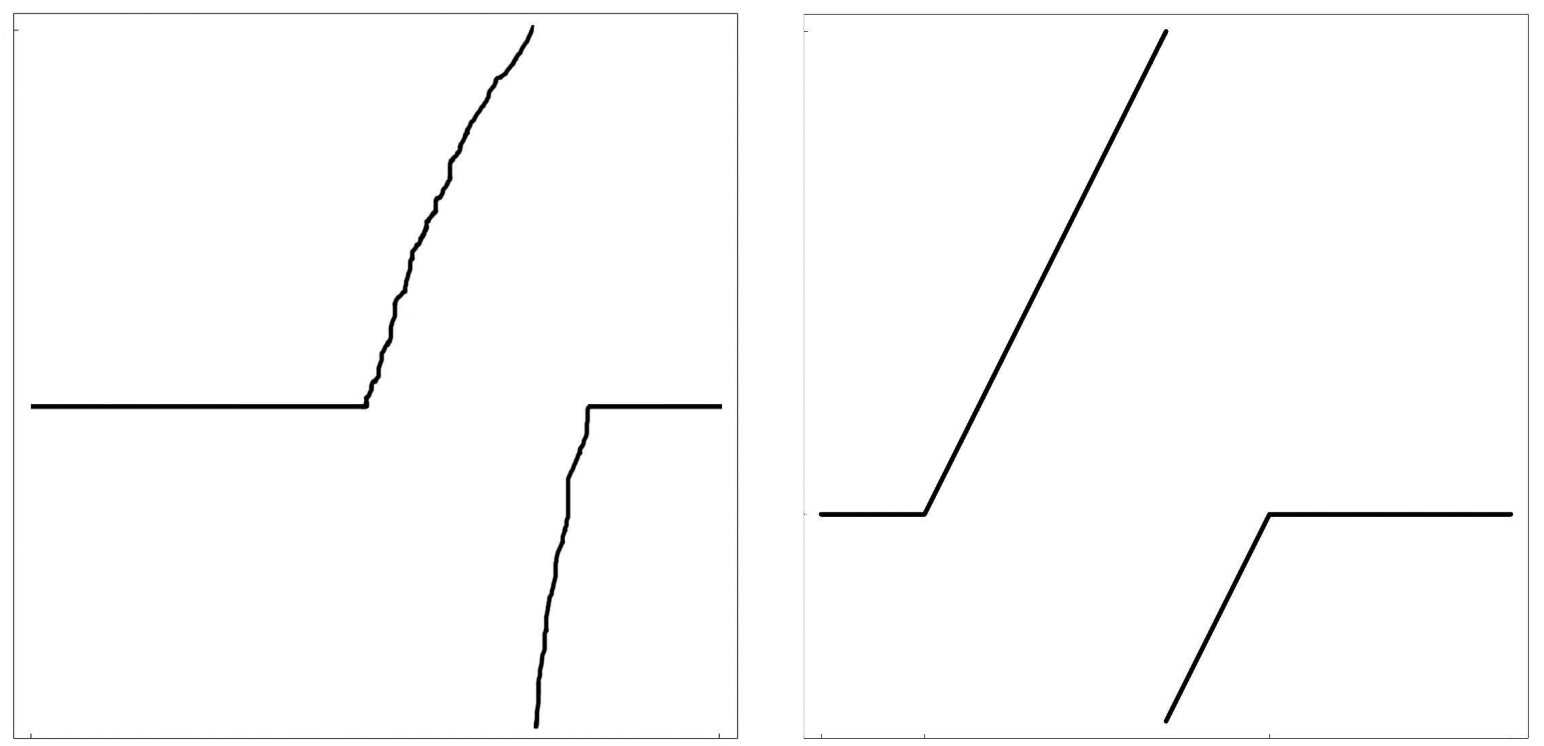

Figure 1. A flat spot map, and the standard flat spot map $T_{3 / 20}$.

The standard family of flat spot maps $T_{\gamma}$ (see e.g. $[4,18,19,20]$ ) is defined for $\gamma \in \mathbb{T}$ by

$$
T_{\gamma}(x)=\left\{\begin{array}{lll}
2 \gamma & (\bmod 1) & \text { for } x \in F_{\gamma}:=(\gamma+1 / 2, \gamma), \\
2 x & (\bmod 1) & \text { for } x \in[\gamma, \gamma+1 / 2]
\end{array}\right.
$$

Maps from this family will be used throughout the article as a concrete illustration of results, and are a particular focus of attention from Section 4 onwards, where we study the way in which the first entrance time functions $e_{\gamma}=e_{T_{\gamma}}$ vary with $\gamma$. Of course as $\gamma$ moves counterclockwise, so too does the support of $e_{\gamma}$, namely the flat spot $F_{\gamma}$. A more subtle understanding of the variation of the mass of $e_{\gamma}$ can be obtained through the study of its median $\omega(\gamma)$, the unique point in $[\gamma, \gamma+1 / 2]$ which divides equally the mass of $e_{\gamma}$ (i.e. satisfies $\int_{\gamma}^{\omega(\gamma)} e_{\gamma}=\int_{\omega(\gamma)}^{\gamma+1 / 2} e_{\gamma}$ ). Computer study of the variation of the point $\omega(\gamma)$ within $F_{\gamma}$ led us to the following surprising discovery ${ }^{1}$ :

Theorem 6.4. Provided $\varrho\left(T_{\gamma}\right) \notin\{0, \pm 2 / 7, \pm 3 / 10, \pm 1 / 3, \pm 3 / 8\}$, the entrance time $e_{\gamma}(\omega(\gamma))$ can only take one of the five values 1, 2, 4, 5, or 12.

\section{Flat SPOT MAPS}

Theorem 2.1. ([2, 4, 19, 21]) Let $T: \mathbb{T} \rightarrow \mathbb{T}$ be a flat spot map. If $\varrho(T)$ is irrational then there exists a totally absorbing $T$-invariant Cantor subset of $\mathbb{T} \backslash F$. If $\varrho(T)$ is rational then either (i) a single periodic orbit is totally absorbing, or (ii) there are precisely two periodic orbits, one of which is absorbing and absorbs all points of $\mathbb{T}$ except those points in the other periodic orbit.

\footnotetext{
${ }^{1}$ See Section 6 for a slightly more detailed statement of Theorem 6.4 .
} 
Proof of Theorem 2.1. If $\varrho(T)$ is irrational then (see e.g. [2, 4, 19, 21]) there is a $T$-invariant Cantor set $K=K_{T}=\cap_{n=0}^{\infty} T^{-n}(\mathbb{T} \backslash F)$, which in particular contains the two endpoints (which we denote by $\alpha$ and $\beta$ ) of $\mathbb{T} \backslash F$. Now $v=T(x)$ for all $x \in F$, and hence for all $x \in \bar{F}$ by continuity of $T$, so $v=T(\alpha)=T(\beta)$ belongs to the Cantor set $K$; thus every point in $F$ is absorbed by $K$. If $x \in(\mathbb{T} \backslash F) \backslash K$ then there exists $n=n(x) \geq 1$ such that $T^{n}(x) \in F\left(\right.$ since $\left.K=\cap_{n=0}^{\infty} T^{-n}(\mathbb{T} \backslash F)\right)$, so $T^{n+1}(x)=v \in K$. So every point in $\mathbb{T} \backslash F$ is absorbed by $K$, and therefore $K$ is totally absorbing.

If $\varrho(T)$ is rational then (see e.g. $[2,4,19,21]$ ) there is a periodic orbit $P$ contained in $\mathbb{T} \backslash F$. First suppose that one ${ }^{2}$ of the endpoints of $\mathbb{T} \backslash F$, denoted $\gamma$, belongs to $P$. Then $T(\gamma)=v$ also lies in $P$. But $T(x)=v$ for all $x \in F$, so $P$ absorbs all points of $F$. Similarly, if $x \in \mathbb{T} \backslash F$ eventually enters $F$ under some $T$-iterate, then it is absorbed by $P$ (on the next iterate after entering $F$ ). Now if $x \in \cap_{n=0}^{\infty} T^{-n}(\mathbb{T} \backslash F)$ then in fact $T^{n}(x) \in P$ for some $n=n(x) \geq 0$ (see e.g. $[2,4,19,21]$ ), so $x$ is absorbed by $P$. Therefore $P$ is totally absorbing.

Next suppose that neither endpoint of $\mathbb{T} \backslash F$ belongs to $P$, in which case (see e.g. $[2,4$, 19, 21]) $P=\cap_{n=0}^{\infty} T^{-n}(\mathbb{T} \backslash F)$. Clearly $v$ does not lie in $P$ (since neither endpoint of $\mathbb{T} \backslash F$ does), so because $P=\cap_{n=0}^{\infty} T^{-n}(\mathbb{T} \backslash F)$ we know there exists $n \geq 0$ such that $T^{n}(v) \in F$, thus $T^{n+1}(v)=v$, therefore $v$ is periodic. Let $Q$ denote the corresponding period- $(n+1)$ orbit $\left\{v, T(v), \ldots, T^{n}(v)\right\}$. Clearly all points in $F$ are absorbed by $Q$. If $x \in(\mathbb{T} \backslash F) \backslash P$ then, since $P=\cap_{n=0}^{\infty} T^{-n}(\mathbb{T} \backslash F)$, we know there is an integer $m=m(x) \geq 1$ such that $T^{m}(x) \in F$, hence $T^{m+1}(x)=v \in Q$, so $x$ is absorbed by $Q$. So $Q$ absorbs all points in $\mathbb{T} \backslash P$, as required.

\section{Remark 2.2.}

(a) We say that the map $T$ is totally absorbing if some proper $T$-invariant subset of $\mathbb{T}$ is totally absorbing. From the proof of Theorem 2.1 we see that if $T$ is a totally absorbing flat spot map, with flat spot $F$, then at least one of the endpoints of $F$ (hence of $\mathbb{T} \backslash F$ ) belongs to the totally absorbing invariant set. More precisely, if the totally absorbing invariant set is a periodic orbit then it contains precisely one endpoint of $F$; if the totally absorbing invariant set is a Cantor set then it contains both endpoints of $F$.

(b) The absorbing invariant set of a flat spot map supports a unique $T$-invariant probability measure $\mu$, which is clearly a physical measure (cf. [9, 22]): for Lebesgue almost every $x \in \mathbb{T}$, the partial orbit measures $n^{-1} \sum_{i=1}^{n-1} \delta_{T^{i} x}$ converge to $\mu$ in the weak-* topology.

\section{EXPLICIT DESCRIPTION OF FIRST ENTRANCE TIMES}

In the case of flat spot maps with rational rotation number, it turns out to be possible to obtain a rather explicit description of the first entrance time function. A key ingredient is the finite partition ${ }^{3}$ of the circle determined by the periodic orbit outside the flat spot:

\footnotetext{
${ }^{2}$ Clearly it is never the case that both endpoints of $\mathbb{T} \backslash F$ are periodic, since they are each mapped by $T$ onto the same point $v$.

${ }^{3}$ By a partition of $\mathbb{T}$ we mean a (finite or countably infinite) collection of intervals whose interiors are pairwise disjoint and whose union is dense in $\mathbb{T}$; as is common in the dynamical systems literature, it is convenient to not insist that the intervals themselves be disjoint.
} 
Definition 3.1. Given a flat spot map $T: \mathbb{T} \rightarrow \mathbb{T}$ with rational rotation number, let $s_{1}<\ldots<s_{q}$ denote its periodic orbit outside $F$. Define its orbit partition to be the partition of $\mathbb{T}$ consisting of the $q$ closed intervals $\left[s_{q}, s_{1}\right],\left[s_{1}, s_{2}\right], \ldots,\left[s_{q-1}, s_{q}\right]$.

The first $q-1$ pre-images of $K_{0}=\left[s_{q}, s_{1}\right]$ under $\left.T\right|_{\mathbb{T} \backslash F}$ are closed sub-intervals in $\mathbb{T} \backslash F$ whose endpoints are consecutive points in the orbit, thus:

Lemma 3.2. Given a flat spot map $T: \mathbb{T} \rightarrow \mathbb{T}$ with rational rotation number, let $s_{1}<$ $\ldots<s_{q}$ denote its periodic orbit outside $F$. The orbit partition consists of intervals $K_{0}, K_{1}, \ldots, K_{q-1}$, with the property that $T$ maps $K_{l}$ onto $K_{l-1}$ for $1 \leq l \leq q-1$.

Example 3.3. If $\gamma \in[9 / 62,5 / 31]$ then the standard flat spot map $T_{\gamma}$ has periodic orbit $\{5 / 31,9 / 31,10 / 31,18 / 31,20 / 31\}$ outside $F_{\gamma}$, and its orbit partition consists of the intervals $K_{0}=[20 / 31,5 / 31], K_{1}=[10 / 31,18 / 31], K_{2}=[5 / 31,9 / 31], K_{3}=[18 / 31,20 / 31], K_{4}=$ $[9 / 31,10 / 31]$.

Definition 3.4. For any flat spot map $T: \mathbb{T} \rightarrow \mathbb{T}$, with flat spot $F$, define the first entrance time function $e_{T}: \mathbb{T} \rightarrow \mathbb{Z}_{\geq 0} \cup\{\infty\}$ by

$$
e_{T}(x)=\inf \left\{i \geq 0: T^{i}(x) \in F\right\} .
$$

The function $e_{T}$ is piecewise constant with respect to some countable partition, as illustrated by the following examples:
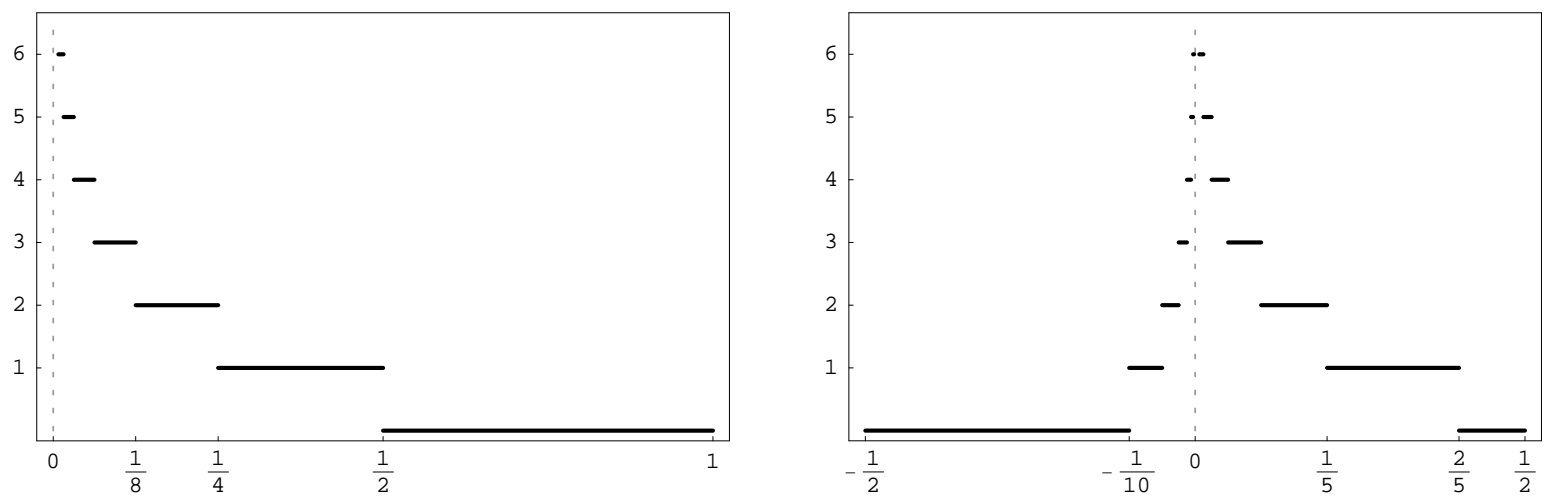

FIGURE 2. Entrance time functions $e_{\gamma}=e_{T_{\gamma}}$ for standard flat spot map $T_{\gamma}$ where $\gamma=0$ and $\gamma=-1 / 10$ (both of rotation number 0 ).

Example 3.5. The standard flat spot map $T=T_{0}$ has rotation number 0 , and totally absorbing fixed point 0 . The entrance time function $e_{T}$ (cf. Figure 2) is given by $e_{T} \equiv n$ on $\left(2^{-n-1}, 2^{-n}\right)$ for $n \geq 0$, and $e_{T} \equiv \infty$ on $\{0\} \cup\left\{2^{-n}: n \geq 1\right\}$.

Example 3.6. For $\gamma \in(1 / 2,1)$, the standard flat spot map $T_{\gamma}$ has rotation number 0 , with fixed point 0 and absorbing fixed point $2 \gamma$. The entrance time function $e_{T}$ (cf. Figure 2 for $\gamma=9 / 10)$ is given by $e_{T} \equiv 0$ on $F_{\gamma}=(\gamma-1 / 2, \gamma)$, and $e_{T} \equiv n$ on $\left(2^{-n}(\gamma-1 / 2), 2^{-n+1}(\gamma-\right.$ $1 / 2)] \cup\left[1+2^{-n+1}(\gamma-1), 1+2^{-n}(\gamma-1)\right)$, and $e_{T}(0)=\infty$.

Example 3.7. The standard flat spot map $T=T_{1 / 6}$ has rotation number $1 / 2$, with totally absorbing period-2 orbit $\{1 / 3,2 / 3\}$. Here (cf. Figure 3 ) $e_{T} \equiv 0$ on $F=(2 / 3,1 / 6$ ), while 

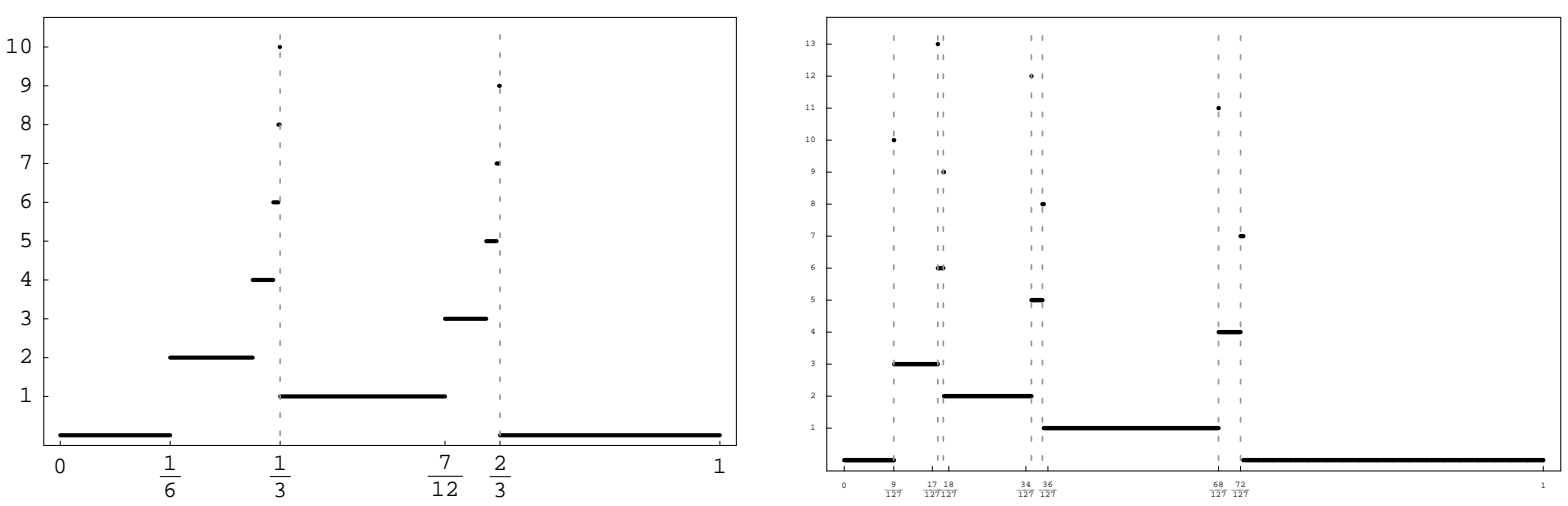

FiguRE 3. Entrance time functions $e_{\gamma}=e_{T_{\gamma}}$ for standard flat spot map $T_{\gamma}$ where $\gamma=1 / 6$ (of rotation number $1 / 2$ ), and $\gamma=9 / 127$ (of rotation number $2 / 7)$.

$e_{T} \equiv 2 m+1$ on $\left(\frac{1}{3}\left(2-4^{-m}\right), \frac{1}{3}\left(2-4^{-m-1}\right)\right)$ for $m \geq 0$, and $e_{T} \equiv 2 m$ on $\left(\frac{1}{6}\left(2-4^{-m+1}\right), \frac{1}{6}(2-\right.$ $\left.4^{-m}\right)$ ) for $m \geq 1$, and $e_{T} \equiv \infty$ on $\{2 / 3\} \cup\left\{\left(2-4^{-m}\right) / 6: m \geq 0\right\} \cup\left\{\left(2-4^{-m}\right) / 3: m \geq 0\right\}$.

Example 3.8. The standard flat spot map $T=T_{1 / 4}$ has rotation number $1 / 2$, with period2 orbit $\{1 / 3,2 / 3\}$ and absorbing period- 2 orbit $\{0,1 / 2\}$. Here $e_{T} \equiv 0$ on $(-1 / 4,1 / 4)=$ $(3 / 4,1 / 4)$, and $e_{T} \equiv 1$ on the interval $(3 / 8,5 / 8)$. We calculate $e_{T} \equiv 2$ on $[1 / 4,5 / 16) \cup$ $(11 / 16,3 / 4]$, and $e_{T} \equiv 3$ on $(11 / 32,3 / 8] \cup[5 / 8,21 / 32)$, and in general for $n \geq 2$ the level set $e_{T}^{-1}(n)$ is a union of two disjoint intervals, its form depending on the parity of $n$ : $e_{T} \equiv 2 m$ on $\left[\frac{1}{3}\left(1-4^{-m}\right), \frac{1}{3}\left(1-4^{-m-1)}\right)\right) \cup\left(\frac{1}{3}\left(2+4^{-m-1)}\right), \frac{1}{3}\left(2+4^{-m}\right)\right]$ and $e_{T} \equiv 2 m+1$ on $\left(\frac{1}{6}\left(2+4^{-m-1}\right), \frac{1}{6}\left(2+4^{-m}\right)\right] \cup\left[\frac{1}{6}\left(4-4^{-m}\right), \frac{1}{6}\left(4-4^{-m-1}\right)\right)$.

Example 3.9. The standard flat spot map $T=T_{5 / 31}$ has rotation number $2 / 5$, with totally absorbing period-5 orbit $\{5 / 31,9 / 31,10 / 31,18 / 31,20 / 31\}$. Here $e_{T} \equiv 0$ on $(41 / 62,5 / 31)$, $e_{T} \equiv 1$ on $(41 / 124,18 / 31), e_{T} \equiv 2$ on $(41 / 248,9 / 31), e_{T} \equiv 3$ on $(289 / 496,20 / 31), e_{T} \equiv 4$ on $(289 / 992,10 / 31)$, and in general for $n \geq 5$ the level set $e_{T}^{-1}(n)$ is an interval, its form depending on the residue class of $n$ modulo $5: e_{T} \equiv 5 m$ on $\left(\frac{1}{31}\left(20+2^{-5 m-1}\right), \frac{1}{31}\left(20+2^{-5 m+4}\right)\right)$, $e_{T} \equiv 5 m+1$ on the interval $\left(\frac{1}{31}\left(10+2^{-5 m-2}\right), \frac{1}{31}\left(10+2^{-5 m+3}\right)\right), e_{T} \equiv 5 m+2$ on the interval $\left(\frac{1}{31}\left(5+2^{-5 m-3}\right), \frac{1}{31}\left(5+2^{-5 m+2}\right)\right), e_{T} \equiv 5 m+3$ on $\left(\frac{1}{31}\left(18+2^{-5 m-4}\right), \frac{1}{31}\left(18+2^{-5 m+1}\right)\right)$, and $e_{T} \equiv 5 m+4$ on $\left(\frac{1}{31}\left(9+2^{-5 m-5}\right), \frac{1}{31}\left(9+2^{-5 m}\right)\right)$.

Notation 3.10. For $0 \leq n \leq \infty$ define $E_{n}=E_{n}(T):=e_{T}^{-1}(n)$, the set of points which first enter $F$ on precisely their $n$th iterate (cf. [5, 8]). The collection $\left\{E_{n}(T)\right\}_{0 \leq n \leq \infty}$ is clearly a partition of $\mathbb{T}$.

Remark 3.11. Features common to Examples 3.5-3.9, and which will turn out to be general phenomena for flat spot maps, are that each level set $E_{n}$ can be written as a union of at most two disjoint intervals, and is a single interval for $n$ sufficiently small. In the cases where $T$ is totally absorbing (Examples 3.5, 3.7 and 3.9), $E_{n}$ is a single interval for all $n$.

These facts will be proved as Theorem 3.13, though the intuitive mechanism by which the two intervals comprising $E_{n}$ are formed (in the non-totally absorbing case) can be 
explained as follows, following closely the suggestion of an anonymous referee. Suppose $T$ maps an interval $I$ so that $T(I) \supset F$, with two sub-intervals $\left(J_{0}\right.$ and $J_{1}$, say) of $T(I)$ protruding from the flat spot $F$, one on either side. Since $T(F)=v$, after one further iteration of $T$ the two intervals which were previously separated by a positive distance are now contiguous (i.e. $T\left(J_{0}\right)$ and $T\left(J_{1}\right)$ share $v$ as a common endpoint). If $T^{n-1}(v) \in F$ then some neighbourhood of $v$ also enters $F$ after $n-1$ iterates, so some sub-interval ( $K_{i}$, say) of each $J_{i}$ enters $F$ after $n$ iterates. The intervals $K_{0}$ and $K_{1}$ are the two components of $E_{n}$.

For flat spot maps with rational rotation number $p / q$, the following Theorem 3.13 gives an explicit description of the entrance time function $e_{T}$ on each orbit partition piece $K_{l}$, $0 \leq l \leq q-1$ (cf. Definition 3.1, Lemma 3.2). First it will be convenient to introduce some notation:

Notation 3.12. Given two non-empty subsets (possibly singletons) $I$ and $I^{\prime}$ of a proper sub-interval $J \subset \mathbb{T}$, we say that $I$ is to the left of $I^{\prime}$, and that $I^{\prime}$ is to the right of $I$, and write $I \prec I^{\prime}$, if $x \leq x^{\prime}$ for all $x \in I, x^{\prime} \in I^{\prime}$, where $\leq$ denotes the ordering on the interval $J$.

Theorem 3.13. Suppose the flat spot map $T: \mathbb{T} \rightarrow \mathbb{T}$ has rational rotation number $p / q$, with periodic orbit $s_{1}<\ldots<s_{q}$ outside $F$. Then for each $0 \leq l \leq q-1$, the interval $K_{l}$ can be written as $K_{l}=\overline{\bigcup_{i \in \mathbb{Z}} E_{l}^{i}}$, where the $E_{l}^{i}$ are pairwise disjoint intervals whose relative ordering in $K_{l}$ is $E_{l}^{i} \prec E_{l}^{i+1}$ for all $i \in \mathbb{Z}$, and $E_{l+i q}=E_{l}^{-i} \cup E_{l}^{i}$ for $i \geq 0$, so in particular $E_{l}=E_{l}^{0}$ is an interval, and $e_{T} \equiv l+|i| q$ on $E_{l}^{i}$ for $i \in \mathbb{Z}$. Moreover, $e_{T} \equiv \infty$ on $E_{\infty} \cap K_{l}=K_{l} \backslash \cup_{i \in \mathbb{Z}} E_{l}^{i}$.

Proof. Let us write $F=(\alpha, \beta)$. It suffices to prove the result when $l=0$, since if $1 \leq$ $l \leq q-1$ then $T^{l}$ maps $K_{l}$ onto $K_{0}$, preserving orientation, and $e_{T}(x)=l+e_{T}\left(T^{l}(x)\right)$ for $x \in K_{l}$, both easy consequences of Lemma 3.2, so the general result follows immediately from the case $l=0$. Suppose $T$ is not totally absorbing (the easier proof for totally absorbing $T$ involves minor modifications and will be omitted). First define $E_{0}^{-}:=\left(s_{q}, \alpha\right]$ and $E_{0}^{+}:=\left[\beta, s_{1}\right)$; we will subsequently introduce intervals $E_{0}^{i}$ for $i \in \mathbb{Z}$, and verify the properties $E_{0}^{-}=\cup_{i=1}^{\infty} E_{0}^{-i}$ and $E_{0}^{+}=\cup_{i=1}^{\infty} E_{0}^{i}$.

Now $E_{0}^{-} \cup E_{0}^{+}$is mapped by $T$ onto the interior of $K_{q-1}$, so Lemma 3.2 implies that $E_{0}^{-} \cup E_{0}^{+}$is mapped by $T^{q}$ onto $\operatorname{int}\left(K_{0}\right)$, which itself contains both $E_{0}^{-}$and $E_{0}^{+}$. Since $T^{q}$ preserves orientation, $T^{q}\left(E_{0}^{-}\right)$(respectively $T^{q}\left(E_{0}^{+}\right)$) is on the left (respectively the right) of $\operatorname{int}\left(K_{0}\right)=T^{q}\left(E_{0}^{-} \cup E_{0}^{+}\right)$with respect to the ordering on $K_{0}$.

Concentrating for the moment on the case of $E_{0}^{+}$, we see that in particular $E_{0}^{+} \subset T^{q}\left(E_{0}^{+}\right)$, so that $T^{-q}\left(E_{0}^{+}\right) \cap[\beta, \alpha]=T^{-q}\left(E_{0}^{+}\right) \cap E_{0}^{+}=: \hat{E}_{0}^{1}$ is a left-closed right-open sub-interval of $E_{0}^{+}$whose right endpoint is $s_{1}$. More generally, for each $i \geq 0$, define $\hat{E}_{0}^{i}:=\cap_{j=0}^{i} T^{-j q}\left(E_{0}^{+}\right)$; in particular, $\hat{E}_{0}^{0}=E_{0}^{+}$. For each $i \geq 0, \hat{E}_{0}^{i}$ is a left-closed right-open sub-interval of $E_{0}^{+}$ whose right endpoint is $s_{1}$; it consists precisely of those points in $E_{0}^{+}$whose first entrance time into $F$ is strictly greater than $i q$ (i.e. at least $(i+1) q$ ).

Define $E_{0}^{0}:=E_{0}$, and for $i \geq 1$ define $E_{0}^{i}:=\hat{E}_{0}^{i-1} \backslash \hat{E}_{0}^{i}$, the set of points in $E_{0}^{+}$whose first entrance time into $F$ is precisely $i q$. The set $E_{0}^{i}$ is a left-closed right-open sub-interval of $E_{0}^{+}$whose left endpoint is the left endpoint of $\hat{E}_{0}^{i-1}$ (in particular, the left endpoint of $E_{0}^{1}$ 
is $\beta$, the left endpoint of $\hat{E}_{0}^{0}=E_{0}^{+}$), and whose right endpoint is the left endpoint of $\hat{E}_{0}^{i}$. Therefore for all $i \geq 1$, the disjoint intervals $E_{0}^{i}$ and $E_{0}^{i+1}$ are adjacent, with $E_{0}^{i} \prec E_{0}^{i+1}$, and $E_{0}^{+}=\cup_{i=1}^{\infty} E_{0}^{i}$. Clearly also $E_{0}^{0}=E_{0} \prec E_{0}^{1}$.

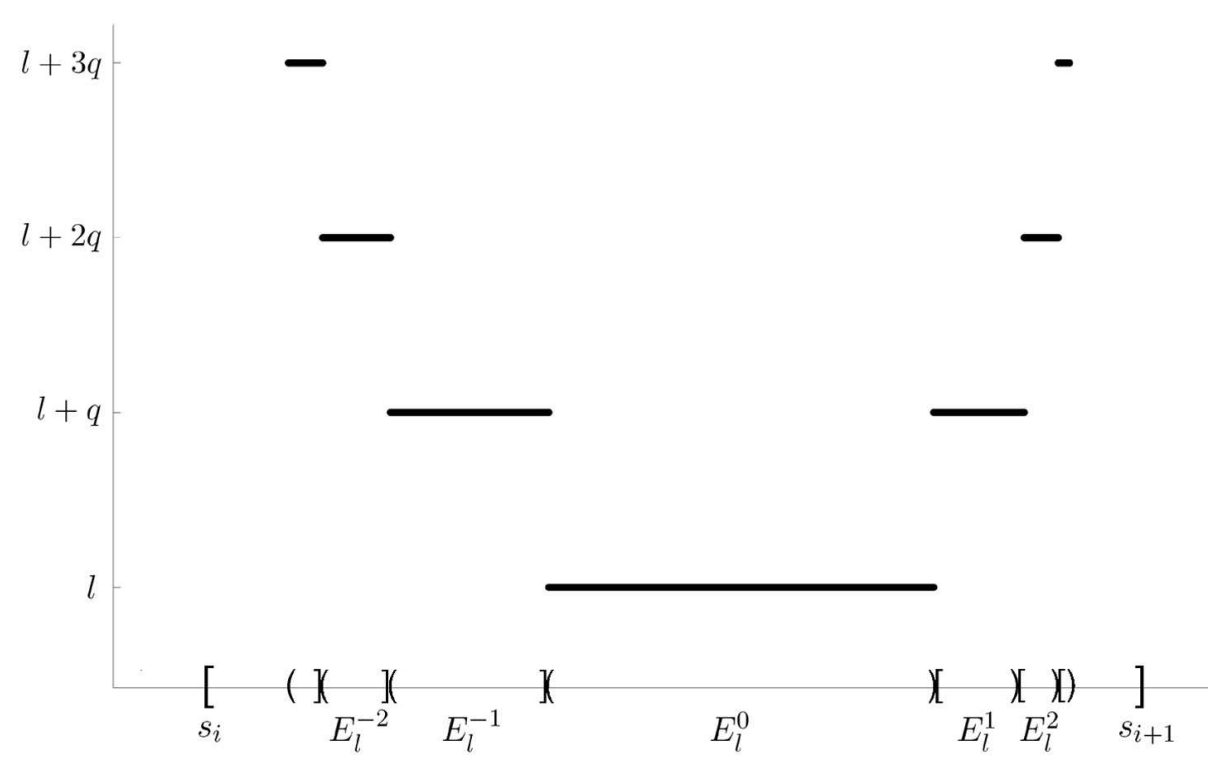

FiguRE 4. Graph of the entrance time function restricted to $K_{l}$

We can argue analogously on the interval $E_{0}^{-}$, defining left-open right-closed intervals $\hat{E}_{0}^{-i}:=\cap_{j=0}^{i} T^{-j q}\left(E_{0}^{-}\right)$and $E_{0}^{-i}:=\hat{E}_{0}^{-(i-1)} \backslash \hat{E}_{0}^{-i}$, with $E_{0}^{-}=\cup_{i=1}^{\infty} E_{0}^{-i}$, and showing that $e_{T} \equiv i q$ on $E_{0}^{-i}$. We have then shown that, for all $i \geq 0, e_{T} \equiv i q$ on $E_{0}^{-i} \cup E_{0}^{i}$, therefore $E_{0}^{-i} \cup E_{0}^{i} \subset E_{i q}$. But the collection $\left\{E_{0}^{j}\right\}_{j \in \mathbb{Z}}$ is a partition of $K_{0}$, so in fact $E_{0}^{-i} \cup E_{0}^{i}=E_{i q}$. Moreover, $\cup_{i \in \mathbb{Z}} E_{0}^{i}=\left(\cup_{i<0} E_{0}^{i}\right) \cup E_{0} \cup\left(\cup_{i>0} E_{0}^{i}\right)=\left(s_{q}, \alpha\right] \cup(\alpha, \beta) \cup\left[\beta, s_{1}\right)=\left(s_{q}, s_{1}\right)=\operatorname{int}\left(K_{0}\right)$. The two endpoints of $K_{0}$ belong to the orbit $\left\{s_{1}, \ldots, s_{q}\right\}$, hence have infinite first entrance time into $F$.

In fact we have proved:

Corollary 3.14. Under the assumptions of Theorem 3.13, if $T$ is not totally absorbing then $E_{l}=E_{l}^{0}$ is an open interval, $E_{l}^{i}$ is a left-open right-closed interval for $i<0$, and a left-closed right-open interval for $i>0$; in this case $\cup_{i \in \mathbb{Z}} E_{l}^{i}=\operatorname{int}\left(K_{l}\right)$, and $E_{\infty} \cap K_{l}$ consists of the two endpoints of $K_{l}$.

Minor modifications of the proof of Theorem 3.13 leads to the following two results in the case of totally absorbing $T$.

Corollary 3.15. Under the assumptions of Theorem 3.13, if $T$ is totally absorbing and the right endpoint of $F$ (i.e. the left endpoint of $\mathbb{T} \backslash F$ ) belongs to the totally absorbing periodic orbit, then $E_{l}^{i}=\emptyset$ for $i<0$, and $E_{l}^{i}=E_{l+i q}$ is a non-empty open interval for $i \geq 0$; in this case $E_{\infty} \cap K_{l}=K_{l} \backslash\left(\cup_{i \in \mathbb{Z}} E_{l}^{i}\right)$ consists of the endpoints of $E_{l}^{i}=E_{l+i q}$ for $i>0$ (these are precisely the points $x \in K_{l}$ which are eventually iterated onto the right endpoint of $F$ ) union the two endpoints of $K_{l}$. 
Corollary 3.16. Under the assumptions of Theorem 3.13, if $T$ is totally absorbing and the left endpoint of $F$ (i.e. the right endpoint of $\mathbb{T} \backslash F$ ) belongs to the totally absorbing periodic orbit, then $E_{l}^{i}=E_{l-i q}$ is a non-empty open interval for $i \leq 0$, and $E_{l}^{i}=\emptyset$ for $i>0$; in this case $E_{\infty} \cap K_{l}=K_{l} \backslash\left(\cup_{i \in \mathbb{Z}} E_{l}^{i}\right)$ consists of the endpoints of $E_{l}^{i}=E_{l-i q}$ for $i<0$ (these are precisely the points $x \in K_{l}$ which are eventually iterated onto the left endpoint of $F$ ) union the two endpoints of $K_{l}$.

Examples 3.5-3.9 can now be seen in the context of the above results:

Example 3.17. For the standard flat spot map $T=T_{0}$ (cf. Example 3.5), the totally absorbing fixed point 0 is the right endpoint of the flat spot, so Corollary 3.15 gives $E_{0}^{i}=\emptyset$ for $i<0$, and $E_{0}^{i}=E_{i}=\left(2^{-i-1}, 2^{-i}\right)$ for $i \geq 0$.

Example 3.18. For $\gamma \in(1 / 2,1)$ (cf. Example 3.6), the standard flat spot map $T_{\gamma}$ is not totally absorbing, so Corollary 3.14 gives $E_{0}^{0}=E_{0}=(\gamma-1 / 2, \gamma)$, and

$$
E_{0}^{i}= \begin{cases}\left(2^{i}(\gamma-1 / 2), 2^{i+1}(\gamma-1 / 2)\right] & \text { for } i<0 \\ {\left[1+2^{-i+1}(\gamma-1), 1+2^{-i}(\gamma-1)\right)} & \text { for } i>0 .\end{cases}
$$

Example 3.19. The standard flat spot map $T=T_{1 / 6}$ (cf. Example 3.7) is totally absorbing, and the left endpoint of the flat spot is the period-2 point $2 / 3$. Its orbit partition consists of parts $K_{0}=[2 / 3,1 / 3]$ and $K_{1}=[1 / 3,2 / 3]$. Corollary 3.16 gives $E_{0}^{0}=E_{0}=$ $(2 / 3,1 / 6) \subset K_{0}$, and $E_{0}^{i}=\emptyset$ for $i>0$, and $E_{0}^{i}=\left(\frac{1}{6}\left(2-4^{i+1}\right), \frac{1}{6}\left(2-4^{i}\right)\right)$ for $i<0$, while $E_{1}^{0}=E_{1}=(1 / 3,7 / 12)$, and $E_{0}^{i}=\emptyset$ for $i>0$, and $E_{1}^{i}=\left(\frac{1}{3}\left(2-4^{i}\right), \frac{1}{3}\left(2-4^{i-1}\right)\right)$ for $i<0$.

Example 3.20. The standard flat spot map $T=T_{1 / 4}$ (cf. Example 3.8) has period-2 orbit $\{1 / 3,2 / 3\}$, and absorbing period-2 orbit $\{0,1 / 2\}$. Its orbit partition consists of parts $K_{0}=[2 / 3,1 / 3]$ and $K_{1}=[1 / 3,2 / 3]$. The interior of $K_{0}$ is the union of the intervals $E_{0}^{0}=E_{0}=(3 / 4,1 / 4)$ and

$$
E_{0}^{i}= \begin{cases}\left(\frac{1}{3}\left(2+4^{i-1}\right), \frac{1}{3}\left(2+4^{i}\right)\right] & \text { for } i<0 \\ {\left[\frac{1}{3}\left(1-4^{-i}\right), \frac{1}{3}\left(1-4^{-i-1)}\right)\right)} & \text { for } i>0,\end{cases}
$$

while the interior of $K_{1}$ is the union of the intervals $E_{1}^{0}=E_{1}=(3 / 8,5 / 8)$ and

$$
E_{1}^{i}= \begin{cases}\left(\frac{1}{6}\left(2+4^{i-1}\right), \frac{1}{6}\left(2+4^{i}\right)\right] & \text { for } i<0 \\ {\left[\frac{1}{6}\left(4-4^{-i}\right), \frac{1}{6}\left(4-4^{-i-1}\right)\right)} & \text { for } i>0 .\end{cases}
$$

Example 3.21. Let $T$ be the standard flat spot map $T_{3 / 20}$, so that $\varrho\left(T_{3 / 20}\right)=2 / 5$, and the periodic orbit outside $F_{3 / 20}$ is $\{5 / 31,9 / 31,10 / 31,18 / 31,20 / 31\}$. For example if $l=2$ then $K_{l}=K_{2}=[5 / 31,9 / 31]$, and $E_{2}=E_{2}^{0}=(13 / 40,23 / 40)$ is a sub-interval of $K_{2}$. For $i \geq 1$, each level set $E_{2+5 i}$ is the union of 2 intervals $E_{2}^{-i}$ and $E_{2}^{i}$. For example: $E_{7}=E_{2}^{-1} \cup E_{2}^{1}$, where $E_{2}^{-1}=(829 / 2560,13 / 40]$ and $E_{2}^{1}=[23 / 40,1479 / 2560) ; E_{12}=E_{2}^{-2} \cup E_{2}^{2}$ where $E_{2}^{-2}=(5305 / 16384,829 / 2560]$ and $E_{2}^{2}=[1479 / 2560,9467 / 16384) ; E_{17}=E_{2}^{-3} \cup E_{2}^{3}$ where $E_{2}^{-3}=(848797 / 2621440,5305 / 16384]$ and $E_{2}^{3}=[9467 / 16384,1514727 / 2621440)$. Note that $E_{2}^{-3} \prec E_{2}^{-2} \prec E_{2}^{-1} \prec E_{2}=E_{2}^{0} \prec E_{2}^{1} \prec E_{2}^{2} \prec E_{2}^{3}$.

Example 3.22. Let $T$ be the (totally absorbing) standard flat spot map $T_{5 / 31}$. As in Example 3.21 , the periodic orbit outside $F_{5 / 31}$ is $\{5 / 31,9 / 31,10 / 31,18 / 31,20 / 31\}$, and 

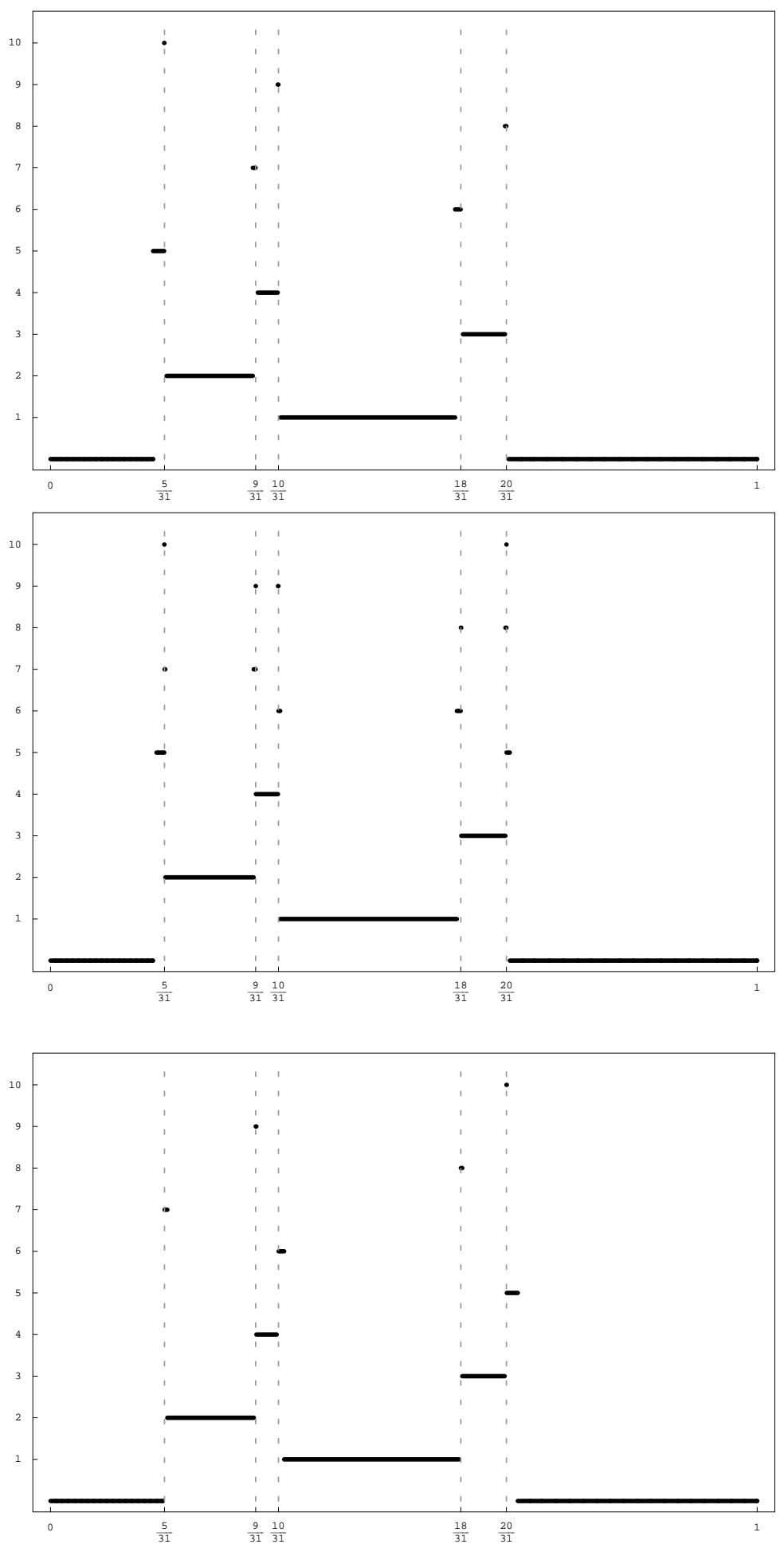

Figure 5. Standard flat spot maps of rotation number 2/5: evolution of entrance time functions $e_{\gamma}=e_{T_{\gamma}}$ as $\gamma$ increases from $\gamma=\gamma_{\min }(2 / 5)=9 / 62$ (top), to $\gamma=3 / 20$ (middle), to $\gamma=\gamma_{\max }(2 / 5)=5 / 31$ (bottom). 
$\varrho\left(T_{5 / 31}\right)=2 / 5$. Then, $E_{1}=E_{1}^{0}=(41 / 124,18 / 31), E_{2}=E_{2}^{0}=(41 / 248,9 / 31), E_{3}=$ $E_{3}^{0}=(289 / 496,20 / 31), E_{4}=E_{4}^{0}=(289 / 992,10 / 31)$, and in general, for $i \geq 1, E_{5 i}=$ $E_{0}^{i}=\left(\frac{1}{31}\left(20+2^{-5 i-1}\right), \frac{1}{31}\left(20+2^{-5 i+4}\right)\right), E_{5 i+1}=E_{1}^{i}=\left(\frac{1}{31}\left(10+2^{-5 i-2}\right), \frac{1}{31}\left(10+2^{-5 i+3}\right)\right)$, $E_{5 i+2}=E_{2}^{i}=\left(\frac{1}{31}\left(5+2^{-5 i-3}\right), \frac{1}{31}\left(5+2^{-5 i+2}\right)\right), E_{5 i+3}=\left(\frac{1}{31}\left(18+2^{-5 i-4}\right), \frac{1}{31}\left(18+2^{-5 i+1}\right)\right)$, and $E_{5 i+4}=E_{4}^{i}=\left(\frac{1}{31}\left(9+2^{-5 i-5}\right), \frac{1}{31}\left(9+2^{-5 i}\right)\right)$. Note that for all $i \geq 1$,

$$
\begin{aligned}
E_{5(i+1)+2} \prec E_{5 i+2} \prec E_{2} & \prec E_{5(i+1)+4} \prec E_{5 i+4} \prec E_{4} \prec E_{5(i+1)+1} \prec E_{5 i+1} \\
& \prec E_{1} \prec E_{5(i+1)+3} \prec E_{5 i+3} \prec E_{3} \prec E_{5(i+1)} \prec E_{5 i} .
\end{aligned}
$$

Remark 3.23. If $T$ has irrational rotation number, the fact that $v=T(F)$ has infinite first entrance time can be used to show that for every $0 \leq n<\infty$, the set $E_{n}(T)$ is a non-empty open interval (i.e. it is connected).

Remark 3.24. As mentioned in Section 1, first entrance time functions have attracted the attention of various authors, though with less emphasis on their fine detail. Notably, the field of open dynamical systems is concerned with a privileged subset $F$ (referred to as a hole $[1,5,7,8,17]$ or a trap [14]) of a phase space of some dynamical system, and the escape (or extinction, cf. [14]) of orbits into $F$. Following the pioneering work of Pianigiani \& Yorke [17], the primary objects of attention are (absolutely continuous) conditionally invariant measures $\mu$ and the escape rate $-\lim _{n \rightarrow \infty} \frac{1}{n} \log \mu\left(\cup_{m>n} E_{m}\right)$, where $E_{m}$ is defined as in Notation 3.10 (for further details see $[1,5,7,8,14,17]$ and references therein). For example if $T$ is any member of the standard family of flat spot maps considered in Section 4 onwards, and the hole $F$ is its flat spot, then the corresponding escape rate equals $1 / 2$, and normalised Lebesgue measure $\mu$ on $\mathbb{T} \backslash F$ is conditionally invariant with eigenvalue $1 / 2$, i.e. $\mu \circ T^{-1}=\frac{1}{2} \mu$.

\section{The STANDARD FAMILY OF FLAT SPOT MAPS}

Recall the standard family $\left(T_{\gamma}\right)_{\gamma \in \mathbb{T}}$ of flat spot maps

$$
T_{\gamma}(x)=\left\{\begin{array}{lll}
2 \gamma & (\bmod 1) & \text { for } x \in F_{\gamma}:=(\gamma+1 / 2, \gamma), \\
2 x & (\bmod 1) & \text { for } x \in[\gamma, \gamma+1 / 2]
\end{array}\right.
$$

All elements of $\mathbb{T}$ are realised as the rotation number of at least one standard flat spot map. In fact, writing $\varrho(\gamma):=\varrho\left(T_{\gamma}\right)$, the map $\gamma \mapsto \varrho\left(T_{\gamma}\right)$ is weakly increasing, of degree 1, and its graph is a devil's staircase (see Figure 6, and [2, 3, 4, 11, 18, 19, 20] for more details): the preimage of any rational rotation number $p / q$ is a positive-length closed interval $^{4} \varrho^{-1}(p / q)=:\left[\gamma_{\min }(p / q), \gamma_{\max }(p / q)\right]$, while the preimage of any irrational is a singleton. Indeed combining Theorem 2.1 with a result proved in (each of) $[2,4,20,21]$ yields:

Proposition 4.1. The set $\left\{\gamma \in \mathbb{T}: T_{\gamma}\right.$ is totally absorbing $\}$ is a Cantor subset of $\mathbb{T}$ with zero Hausdorff dimension. In particular, $\{\gamma \in \mathbb{T}: \varrho(\gamma) \notin \mathbb{Q}\}$ has zero Hausdorff dimension, and hence zero Lebesgue measure.

\footnotetext{
${ }^{4}$ In the context of parametrised families of circle homeomorphisms, the phenomenon of the rotation number 'sticking' at rational values as parameters are varied is known as 'frequency locking' or 'mode locking' (see e.g. [3, 11, 15]). The family $\left(T_{\gamma}\right)$ enjoys a stronger form of locking, however: the same set $\left\{s_{1}, \ldots, s_{q}\right\}$ is a $T_{\gamma}$-periodic orbit for every $\gamma \in\left[\gamma_{\min }(p / q), \gamma_{\max }(p / q)\right]$, since the restriction to $\left[s_{1}, s_{q}\right]$ of all these $T_{\gamma}$ is identical.
} 


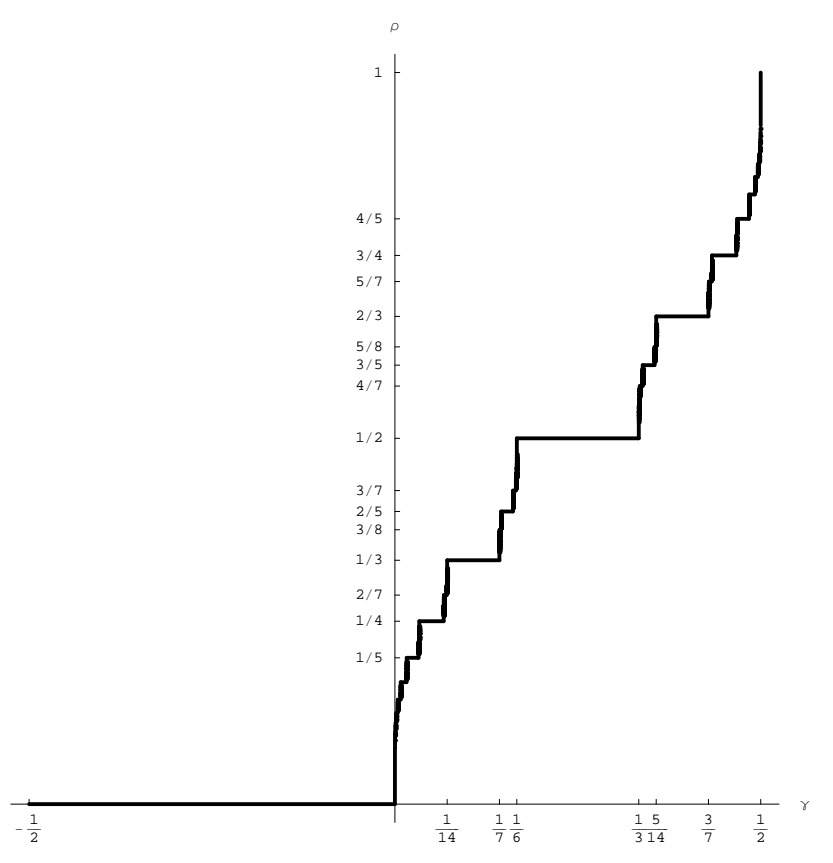

Figure 6. The devil's staircase $\gamma \mapsto \varrho(\gamma)=\varrho\left(T_{\gamma}\right)$.

If $T_{\gamma}$ has rotation number $p / q$, with periodic orbit $s_{1}<\ldots<s_{q}$ outside $F_{\gamma}$, then the largest piece $K_{0}=\left[s_{q}, s_{1}\right]$ of the orbit partition is easily seen to be of length $2^{q-1}\left(2^{q}-\right.$ $1)^{-1}$ (see also [4, Prop. 3]), and the following refinement of Lemma 3.2 is easily proved (cf. Example 3.3):

Lemma 4.2. If $T_{\gamma}$ has rotation number $p / q$, then the orbit partition consists of intervals $K_{0}, K_{1}, \ldots, K_{q-1}$, where each $K_{l}$ has length $2^{q-1-l}\left(2^{q}-1\right)^{-1}$, with the property that $T_{\gamma}$ maps $K_{l}$ affinely onto $K_{l-1}$ for $1 \leq l \leq q-1$.

For $0 \leq n<\infty$, as the $n$-th pre-image of the length- $1 / 2$ interval $E_{0}(\gamma)=F_{\gamma}=(\gamma+$ $1 / 2, \gamma)$ under $\left.T\right|_{[\gamma, \gamma+1 / 2]}$, the set $E_{n}(\gamma)$ has Lebesgue measure ${ }^{5}\left|E_{n}(\gamma)\right|=1 / 2^{n+1}$. Thus $\sum_{n=0}^{\infty}\left|E_{n}(\gamma)\right|=1$, and $\left|E_{\infty}(\gamma)\right|=0$. Since $e_{\gamma}:=e_{T_{\gamma}}=\sum_{n=0}^{\infty} n \chi_{E_{n}(\gamma)}$ Lebesgue almost everywhere, we observe that each first entrance time function $e_{\gamma}$ is Lebesgue integrable, with $^{6} \int e_{\gamma}=1$. In particular, $e_{\gamma}$ is the Radon-Nikodym derivative of a probability measure on $\mathbb{T}$ which is absolutely continuous with respect to Lebesgue measure; we write $|A|_{\gamma}=$ $\int_{A} e_{\gamma}$ to denote the mass of a Borel set $A$ with respect to this measure, and refer to this quantity as the $e_{\gamma}$-mass of $A$.

For the map $T_{\gamma}$ we write $E_{l}(\gamma)$ and $E_{l}^{i}(\gamma)$ to denote $E_{l}$ and $E_{l}^{i}$, and set $E_{l}^{-}(\gamma):=$ $\cup_{i=1}^{\infty} E_{l}^{-i}(\gamma)$ and $E_{l}^{+}(\gamma):=\cup_{i=1}^{\infty} E_{l}^{i}(\gamma)$. We then have the following result, a quantitative complement to Theorem 3.13 which will be exploited in our subsequent study of the entrance time median.

\footnotetext{
${ }^{5}$ The Lebesgue measure of a set $I$ will be denoted by $|I|$; of course if $I$ is an interval then $|I|$ is its length.

${ }^{6}$ We use $\int g$ to denote the Lebesgue integral of a function $g$ on the whole circle $\mathbb{T}$, and $\int_{A} g$ or $\int_{a}^{b} g$ for its Lebesgue integral over the set $A$ or interval $[a, b]$.
} 
Theorem 4.3. If $T_{\gamma}$ has rotation number $p / q$, with periodic orbit $s_{1}<\ldots<s_{q}$ outside $F_{\gamma}$, then for each $0 \leq l \leq q-1$,

(a) $E_{l}(\gamma)=E_{l}^{0}(\gamma)$ has length $2^{-l-1}$, and constitutes proportion ${ }^{7} 1-2^{-q}$ of $K_{l}$. The set $E_{l}^{-}(\gamma)$ has Lebesgue measure $2^{-l}\left(\gamma+1 / 2-s_{q}\right)$, and constitutes proportion $2\left(\gamma+1 / 2-s_{q}\right)(1-$ $\left.2^{-q}\right)$ of $K_{l}$. The set $E_{l}^{+}(\gamma)$ has Lebesgue measure $2^{-l}\left(s_{1}-\gamma\right)$, and constitutes proportion $2\left(s_{1}-\gamma\right)\left(1-2^{-q}\right)$ of $K_{l}$.

(b) For $i \geq 1$, the interval $E_{l}^{-i}(\gamma)$ (respectively $E_{l}^{i}(\gamma)$ ) constitutes proportion $2^{-q i}\left(2^{q}-1\right)$ of $E_{l}^{-}(\gamma)$ (respectively $E_{l}^{+}(\gamma)$ ).

Proof. As in Theorem 3.13, it suffices to prove the result when $l=0$, since if $1 \leq l \leq q-1$ then $T^{l}$ maps $K_{l}$ affinely onto $K_{0}$, preserving orientation, and $e_{\gamma}(x)=l+e_{\gamma}\left(T^{l}(x)\right)$ for $x \in K_{l}$. As before we focus on the case where $T_{\gamma}$ is not totally absorbing (the totally absorbing case requiring only minor amendments). The interval $E_{0}(\gamma)=F_{\gamma}=(\gamma+1 / 2, \gamma)$ has length $1 / 2$, and $K_{0}$ has length $2^{q-1}\left(2^{q}-1\right)^{-1}$, thus $E_{0}(\gamma)$ constitutes proportion $1-2^{-q}$ of $K_{0}$.

Let $\lambda_{q}:=\frac{1}{2}\left(2^{q}-1\right)^{-1}$. Now the interval $E_{0}^{-}(\gamma)$ has length $\gamma+1 / 2-s_{q}$, and constitutes proportion $\left(\gamma+1 / 2-s_{q}\right) / \lambda_{q}$ of the length- $\lambda_{q}$ set $K_{0} \backslash E_{0}(\gamma)$, which itself constitutes proportion $2^{-q}$ of $K_{0}$. It follows that $E_{0}^{-}(\gamma)$ constitutes proportion $2^{-q}\left(\gamma+1 / 2-s_{q}\right) / \lambda_{q}=$ $2\left(\gamma+1 / 2-s_{q}\right)\left(1-2^{-q}\right)$ of $K_{0}$. Similarly $E_{0}^{+}(\gamma)$ has length $s_{1}-\gamma$, and constitutes proportion $2\left(s_{1}-\gamma\right)\left(1-2^{-q}\right)$ of $K_{0}$.

As noted in the proof of Theorem 3.13, $\hat{E}_{0}^{1}(\gamma)=T^{-q}\left(E_{0}^{+}(\gamma)\right) \cap[\gamma, \gamma+1 / 2]=T^{-q}\left(E_{0}^{+}(\gamma)\right) \cap$ $E_{0}^{+}(\gamma)$ is a left-closed right-open sub-interval of $E_{0}^{+}(\gamma)$ whose right endpoint is $s_{1}$; its length is $2^{-q}$ times that of $E_{0}^{+}(\gamma)$. More generally for each $i \geq 0, \hat{E}_{0}^{i}(\gamma)$ is a left-closed right-open sub-interval of $E_{0}^{+}(\gamma)$ whose right endpoint is $s_{1}$; its length is $2^{-i q}$ times that of $E_{0}^{+}(\gamma)$. So for each $i \geq 1, \hat{E}_{0}^{i}(\gamma)$ constitutes proportion $2^{-q}$ of $\hat{E}_{0}^{i-1}(\gamma)$, and $E_{0}^{i}(\gamma)$ constitutes proportion $1-2^{-q}$ of $\hat{E}_{0}^{i-1}(\gamma)$. It follows that $E_{0}^{i}(\gamma)$ constitutes proportion $2^{-(i-1) q}\left(1-2^{-q}\right)=2^{-q i}\left(2^{q}-1\right)$ of $\hat{E}_{0}^{0}(\gamma)=E_{0}^{+}(\gamma)$.

A consequence is the following formula for the average, taken over any orbit partition piece $K_{l}$, of the time of first entrance in $F_{\gamma}$; perhaps surprisingly, this average only depends on (the denominator of) the rotation number of $T_{\gamma}$.

Corollary 4.4. If $T_{\gamma}$ has rational rotation number $p / q$, then

$$
\left|K_{l}\right|_{\gamma}=\frac{2^{q-1-l}}{2^{q}-1}\left(l+\frac{q}{2^{q}-1}\right) \quad \text { for each } \quad 0 \leq l \leq q-1 .
$$

In other words, the conditional expectation ${ }^{8} \mathbb{E}\left(e_{\gamma} \mid\left\{K_{l}\right\}\right)$ is the function which is constant on each partition piece $K_{l}$, with $\left.\mathbb{E}\left(e_{\gamma} \mid\left\{K_{l}\right\}\right)\right|_{K_{l}}=\left|K_{l}\right|_{\gamma} /\left|K_{l}\right|=l+\frac{q}{2^{q}-1}$ for each $0 \leq l \leq q-1$.

Proof. By Theorem 4.3, for each $i \geq 0$, proportion $2^{-i q}\left(1-2^{-q}\right)$ of the points in $K_{l}$ first enter $F_{\gamma}=(\gamma+1 / 2, \gamma)$ after exactly $l+i q$ iterates, so the mean first entrance time is

$$
\frac{\left|K_{l}\right|_{\gamma}}{\left|K_{l}\right|}=\sum_{i=0}^{\infty}(l+i q) 2^{-i q}\left(1-2^{-q}\right)=l+\frac{q}{2^{q}-1} .
$$

\footnotetext{
${ }^{7}$ For $\xi \in[0,1]$, and Borel subsets $A, B \subset \mathbb{T}$, we say $A$ constitutes proportion $\xi$ of $B$ if $|A|=\xi|B|$.

${ }^{8}$ To be precise, we mean the conditional expectation relative to the algebra generated by the orbit partition $\left\{K_{l}\right\}_{l=0}^{q-1}$, where the underlying measure is Lebesgue measure.
} 
Each $K_{l}$ has length $2^{q-1-l}\left(2^{q}-1\right)^{-1}$, so (2) follows.

Example 4.5. For any $\gamma \in \varrho^{-1}(2 / 5)=[9 / 62,5 / 31]$, if $l=2$ then $K_{l}=K_{2}=[5 / 31,9 / 31]$, so $\left|K_{2}\right|_{\gamma} /\left|K_{2}\right|=2+5 /\left(2^{5}-1\right)=67 / 31$, and $\left|K_{2}\right|_{\gamma}=\frac{2^{2}}{2^{5}-1}\left(2+\frac{5}{2^{5}-1}\right)=\frac{268}{961}$.

Corollary 4.6. If $T_{\gamma}$ has rational rotation number $p / q$, and periodic orbit $s_{1}<\ldots<s_{q}$ outside $F_{\gamma}$, then for any $0 \leq l \leq q-1, i \geq 1$,

$$
\left|E_{l}^{i}(\gamma)\right|_{\gamma}=(l+i q) 2^{-(l+i q)}\left(2^{q}-1\right)\left(s_{1}-\gamma\right)
$$

and

$$
\left|E_{l}^{-i}(\gamma)\right|_{\gamma}=(l+i q) 2^{-(l+i q)}\left(2^{q}-1\right)\left(\gamma+1 / 2-s_{q}\right)
$$

so for all $j \geq 0$,

$$
\sum_{i=j+1}^{\infty}\left|E_{l}^{i}(\gamma)\right|_{\gamma}=\frac{s_{1}-\gamma}{2^{l+j q}}\left(l+j q+\frac{q}{1-2^{-q}}\right)
$$

and

$$
\sum_{i=j+1}^{\infty}\left|E_{l}^{-i}(\gamma)\right|_{\gamma}=\frac{\gamma+1 / 2-s_{q}}{2^{l+j q}}\left(l+j q+\frac{q}{1-2^{-q}}\right),
$$

and in particular

$$
\left|E_{l}^{+}(\gamma)\right|_{\gamma}=\frac{s_{1}-\gamma}{2^{l}}\left(l+\frac{q}{1-2^{-q}}\right)
$$

and

$$
\left|E_{l}^{-}(\gamma)\right|_{\gamma}=\frac{\gamma+1 / 2-s_{q}}{2^{l}}\left(l+\frac{q}{1-2^{-q}}\right) .
$$

Proof. Now $e_{\gamma} \equiv l+i q$ on $E_{l}^{i}(\gamma)$, so

$$
\begin{aligned}
\left|E_{l}^{i}(\gamma)\right|_{\gamma} & =(l+i q)\left|E_{l}^{i}(\gamma)\right|=(l+i q) 2^{-q i}\left(2^{q}-1\right) 2^{-l}\left(s_{1}-\gamma\right) \\
& =(l+i q) 2^{-(l+i q)}\left(2^{q}-1\right)\left(s_{1}-\gamma\right)
\end{aligned}
$$

and (4) is proved analogously. The sums (5) and (6) are short calculations, while (7) and (8) follow by setting $j=0$.

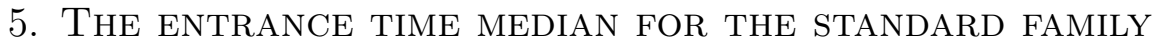

Let $T_{\gamma}$ be a standard flat spot map with rational rotation number $p / q$. From Theorem 3.13 we have an explicit description of the first entrance time function $e_{\gamma}=e_{T_{\gamma}}$ on each orbit partition piece $K_{l}, 0 \leq l \leq q-1$. Since the $K_{l}$ constitute a partition of $\mathbb{T}$, this yields a complete description of $e_{\gamma}$ on the whole of $\mathbb{T}$, provided we understand the relative locations of the orbit partition pieces $K_{l}$. For this we require the following:

Definition 5.1. If $T_{\gamma}$ has rotation number $p / q$, with periodic orbit $s_{1}<\ldots<s_{q}$ outside $F_{\gamma}$, then define $J_{i}:=\left[s_{i}, s_{i+1}\right]$ for $1 \leq i \leq q-1$. So $\left\{J_{i}\right\}_{i=1}^{q-1}$ and $\left\{K_{l}\right\}_{l=1}^{q-1}$ are different indexings of the same collection of intervals, namely the orbit partition with the largest piece removed, and if the permutation $\pi_{p, q}:\{1,2, \ldots, q-1\} \rightarrow\{1,2, \ldots, q-1\}$ is defined by $\pi_{p, q}(l):=-l p(\bmod q)$, then $K_{l}=J_{\pi_{p, q}(l)}$ for $1 \leq l \leq q-1$. 


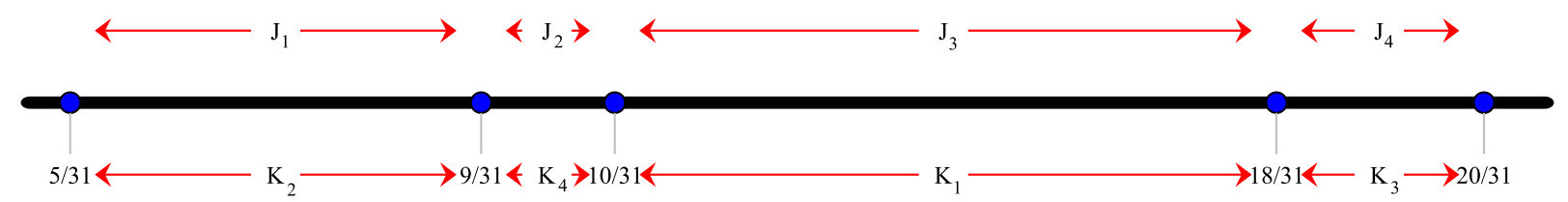

FiguRE 7 . Orbit partition when $T_{\gamma}$ has rotation number $2 / 5$

Example 5.2. If $\gamma \in \varrho^{-1}(2 / 5)=[9 / 62,5 / 31]$ then $J_{1}=[5 / 31,9 / 31], J_{2}=[9 / 31,10 / 31]$, $J_{3}=[10 / 31,18 / 31], J_{4}=[18 / 31,20 / 31]$, so $K_{1}=J_{3}, K_{2}=J_{1}, K_{3}=J_{4}, K_{4}=J_{2}$ (cf. Figure 7).

Notation 5.3. For $\gamma \in \varrho^{-1}(p / q), 1 \leq l \leq q-1, i \in \mathbb{Z}$, let $\epsilon_{l}^{i}(\gamma)$ denote the left endpoint of $E_{l}^{i}(\gamma)$, so that, by Theorem $3.13, \epsilon_{l}^{i+1}(\gamma)$ is the right endpoint of $E_{l}^{i}(\gamma)$.

Definition 5.4. Since $e_{\gamma}$ is strictly positive on $\mathbb{T} \backslash F_{\gamma}=[\gamma, \gamma+1 / 2]$, with $\int_{\gamma}^{\gamma+1 / 2} e_{\gamma}=$ $\sum_{n \geq 1} n\left|E_{n}(\gamma)\right|=\sum_{n \geq 1} n / 2^{n+1}=1$, there is a unique $\omega(\gamma) \in[\gamma, \gamma+1 / 2]$ such that

$$
\int_{\gamma}^{\omega(\gamma)} e_{\gamma}=1 / 2=\int_{\omega(\gamma)}^{\gamma+1 / 2} e_{\gamma} .
$$

The value $\omega(\gamma)$ will be referred to as the entrance time median of $T_{\gamma}$.

Example 5.5. The entrance time median of the standard flat spot map $T_{0}$ is $\omega(0)=1 / 8$, since (cf. Example 3.5) $\int_{1 / 8}^{1 / 2} e_{0}=|[1 / 8,1 / 4]|_{0}+|[1 / 4,1 / 2]|_{0}=2 \times \frac{1}{8}+1 \times \frac{1}{4}=1 / 2$.

Example 5.6. When $\gamma=3 / 4$, the function $e_{\gamma}=e_{3 / 4}$ is symmetric about the point 0 (cf. Example 3.6), so $\omega(3 / 4)=0$.

Example 5.7. For $\gamma \in(3 / 4,1)$, a computation using the entrance time function described in Example 3.6 yields that, for $n \geq 3$, if $\gamma_{n}:=\frac{1}{2}+\frac{1}{4-2^{2-n}-2^{1-n} n}$, then

$$
\omega(\gamma)=\frac{1}{n}\left(2\left(1-2^{-n}\right) \gamma+2^{-n}-3 / 2\right) \quad \text { for } \gamma \in\left[\gamma_{n}, \gamma_{n-1}\right]
$$

while for $\gamma \in(1 / 2,3 / 4)$ the identity $e_{\gamma}(x)=e_{\frac{3}{2}-\gamma}(1-x)$ yields $\omega(\gamma)=1-\omega\left(\frac{3}{2}-\gamma\right)$ (i.e. skew symmetry of the graph relative to the point $(\gamma, \omega)=(3 / 4,0))$, so $\left.\omega\right|_{(1 / 2,3 / 4)}$ is also determined by (10).

Example 5.8. When $\gamma=1 / 4$, the function $e_{\gamma}=e_{1 / 4}$ is symmetric about the point $1 / 2$ (cf. Example 3.8), so $\omega(1 / 4)=1 / 2$.

The above calculations are ad hoc, all relying on some simplifying feature in order to obtain $\omega(\gamma)$. In general the computation of $\omega(\gamma)$ is significantly more difficult, however, and our aim now is to develop an efficient method of locating it. The first step is the following technical lemma.

Lemma 5.9. For rotation number $p / q$, and any $1 \leq l \leq q-1, i \in \mathbb{Z}$, the function $\gamma \mapsto\left|\left[\gamma, \epsilon_{l}^{i}(\gamma)\right]\right|_{\gamma}$ is affine decreasing on $\varrho^{-1}(p / q)$, with slope $\frac{l-i q}{2^{l-i q}}-\frac{q}{1-2^{-q}}\left(1-2^{-(l-i q)}\right)$ if $i \leq 0$, and slope $\frac{l+i q}{2^{l+i q-q}}-\frac{q}{1-2^{-q}}\left(1-2^{-(l+i q)}\right)$ if $i>0$. 
Proof. If $i \leq 0$, then $\left[\gamma, \epsilon_{l}^{i}(\gamma)\right]=E_{0}^{+}(\gamma) \cup\left[s_{1}, s_{\pi_{p, q}(l)}\right] \cup \bigcup_{k=-\infty}^{i-1} E_{l}^{k}(\gamma)$, so

$$
\begin{aligned}
& \left|\left[\gamma, \epsilon_{l}^{i}(\gamma)\right]\right|_{\gamma}=\left|E_{0}^{+}(\gamma)\right|_{\gamma}+\sum_{i=1}^{\pi_{p, q}(l)-1}\left|K_{\pi_{p, q}^{-1}(i)}\right|_{\gamma}+\sum_{k=-\infty}^{i-1}\left|E_{l}^{k}(\gamma)\right|_{\gamma} \\
& \quad=\left(s_{1}-\gamma\right) \frac{q}{1-2^{-q}}+\sum_{i=1}^{\pi_{p, q}(l)-1}\left|K_{\pi_{p, q}^{-1}(i)}\right|_{\gamma}+\frac{\gamma+1 / 2-s_{q}}{2^{l-i q}}\left(l-i q+\frac{q}{1-2^{-q}}\right),
\end{aligned}
$$

using (6), (7), and the result follows because $\sum_{i=1}^{\pi_{p, q}(l)-1}\left|K_{\pi_{p, q}^{-1}(i)}\right|_{\gamma}$ is independent of $\gamma$, by Corollary 4.4. The calculation is analogous when $i \geq 0$ : in this case we write

$$
\left|\left[\gamma, \epsilon_{l}^{i}(\gamma)\right]\right|_{\gamma}=\left|E_{0}^{+}(\gamma)\right|_{\gamma}+\left|\left[s_{1}, s_{\pi_{p, q}(l)+1}\right]\right|_{\gamma}-\sum_{k=i}^{\infty}\left|E_{l}^{k}(\gamma)\right|_{\gamma},
$$

then use (5), (7), and that $\gamma \mapsto\left|\left[s_{1}, s_{\pi_{p, q}(l)}\right]\right|_{\gamma}$ is constant, by Corollary 4.4.

Example 5.10. The function $\gamma \mapsto\left|\left[\gamma, \epsilon_{4}^{1}(\gamma)\right]\right|_{\gamma}$ is affine decreasing on the interval $\varrho^{-1}(2 / 5)=$ $[9 / 62,5 / 31]$, with slope $9 / 2^{9-5}-5\left(1-2^{-9}\right) /\left(1-2^{-5}\right)=-569 / 124 \approx-4.59$.

Example 5.11. Let $\gamma=\gamma_{\min }(2 / 5)=9 / 62$. Then $\left|\left[\gamma, \epsilon_{4}^{1}(\gamma)\right]\right|_{\gamma}=\left[\left.E_{0}^{+}(\gamma)\right|_{\gamma}+\left|K_{2}\right|_{\gamma}+\left|E_{4}\right|_{\gamma}=\right.$ $\left[\left.E_{0}(\gamma)\right|_{\gamma}+\left|K_{2}\right|_{\gamma}+\left|E_{4}\right|_{\gamma}=\frac{2^{5} .5}{2\left(2^{5}-1\right)^{2}}+\left|\left[s_{1}, s_{2}\right]\right|_{\gamma}+\left|E_{4}\right|_{\gamma}=\frac{80}{961}+\frac{2^{5-1-2}}{2^{5}-1}\left(2+\frac{5}{2^{5}-1}\right)+4 / 2^{5}=\right.$ $80 / 961+4.67 / 31^{2}+4 / 2^{5}=3745 / 7688$.

Now let $\gamma=\gamma_{\max }(2 / 5)=5 / 31$. Then $\left|\left[\gamma, \epsilon_{4}^{1}(\gamma)\right]\right|_{\gamma}=4.67 / 31^{2}+129 / 31^{2}=397 / 31^{2}=$ $397 / 961$. By Example 5.10, we deduce that if $\gamma=3 / 20$ then $\left|\left[\gamma, \epsilon_{4}^{1}(\gamma)\right]\right|_{\gamma}=\frac{143}{124}-\frac{569}{124} \frac{3}{20}=$ $1153 / 2480$.

\section{LOCATING THE ENTRANCE TIME MEDiAN}

The aim of this final section is to describe a surprising phenomenon concerning the location of the entrance time median $\omega(\gamma)$ relative to the partition $\left\{E_{n}(\gamma)\right\}_{0 \leq n \leq \infty}$. Our first step is the following consequence of Lemma 5.9:

Lemma 6.1. For rational rotation number $p / q$, and $1 \leq l \leq q-1, i \in \mathbb{Z}$, suppose that $\gamma_{1}, \gamma_{2} \in \varrho^{-1}(p / q)$ satisfy $\omega\left(\gamma_{1}\right) \in \overline{E_{l}^{i}\left(\gamma_{1}\right)}$ and $\omega\left(\gamma_{2}\right) \in \overline{E_{l}^{i}\left(\gamma_{2}\right)}$. Then $\omega(\gamma) \in E_{l}^{i}(\gamma)$ for all $\gamma \in\left(\gamma_{1}, \gamma_{2}\right)$

Proof. Define $f_{\gamma}(t):=|[\gamma, t]|_{\gamma}-1 / 2$, for $\gamma \in\left[\gamma_{1}, \gamma_{2}\right], t \in[\gamma, \gamma+1 / 2]$; the unique zero of $f_{\gamma}$ is the entrance time median $\omega(\gamma)$. From Lemma 5.9,

$$
\gamma \mapsto f_{\gamma}\left(\epsilon_{l}^{i}(\gamma)\right) \text { is decreasing on }\left[\gamma_{1}, \gamma_{2}\right] \text {, }
$$

and

$$
\gamma \mapsto f_{\gamma}\left(\epsilon_{l}^{i+1}(\gamma)\right) \text { is decreasing on }\left[\gamma_{1}, \gamma_{2}\right] \text {. }
$$

For $j=1,2$, the function $f_{\gamma_{j}}$ is increasing, since $e_{\gamma_{j}} \geq 0$, and the zero $\omega\left(\gamma_{j}\right)$ of $f_{\gamma_{j}}$ lies in $\overline{E_{l}^{i}\left(\gamma_{j}\right)}$, an interval whose left endpoint is $\epsilon_{l}^{i}\left(\gamma_{j}\right)$ and whose right endpoint is $\epsilon_{l}^{i+1}\left(\gamma_{j}\right)$. Therefore

$$
f_{\gamma_{j}}\left(\epsilon_{l}^{i}\left(\gamma_{j}\right)\right) \leq 0 \quad \text { and } \quad f_{\gamma_{j}}\left(\epsilon_{l}^{i+1}\left(\gamma_{j}\right)\right) \geq 0 \quad \text { for } j=1,2
$$

If $\gamma \in\left(\gamma_{1}, \gamma_{2}\right)$ then (11) and (13) imply that

$$
f_{\gamma}\left(\epsilon_{l}^{i}(\gamma)\right)<f_{\gamma_{1}}\left(\epsilon_{l}^{i}\left(\gamma_{1}\right)\right) \leq 0
$$


while (12) and (13) imply that

$$
f_{\gamma}\left(\epsilon_{l}^{i+1}(\gamma)\right)>f_{\gamma_{2}}\left(\epsilon_{l}^{i+1}\left(\gamma_{2}\right)\right) \geq 0
$$

But $f_{\gamma}$ is an increasing function, so (14) and (15) imply that its unique zero $\omega(\gamma)$ lies in $E_{l}^{i}(\gamma)$, as required.

If $A$ and $A^{\prime}$ are any two subsets of an interval $J$, we say that $A$ is weakly to the left (respectively the right) of $A^{\prime}$ if there exists $a^{\prime} \in A^{\prime}$ such that $a \leq a^{\prime}$ for all $a \in A$ (respectively $a^{\prime} \leq a$ for all $a \in A$ ). The following obvious lemma will be used systematically, and without comment, to prove Theorem 6.4 below.

Lemma 6.2. Let $\gamma \in \mathbb{T}$. For $n \in \mathbb{N}$, if the $e_{\gamma}$-mass of the interval consisting of those points of $[\gamma, \gamma+1 / 2]$ weakly to the left (respectively the right) of $E_{n}(\gamma)$ is strictly larger than $1 / 2$, then the entrance time median $\omega(\gamma)$ is weakly to the left (respectively the right) of $E_{n}(\gamma)$.

A simple consequence is the following information on the approximate location of $\omega(\gamma)$.

Lemma 6.3. If $\gamma \in \varrho^{-1}(0,1 / 2)$ then $\omega(\gamma)$ is weakly to the right of $E_{2}(\gamma)$ and weakly to the left of $E_{1}(\gamma)$.

Proof. Since $\gamma \in \varrho^{-1}(0,1 / 2)$, both $E_{1}(\gamma)=(\gamma / 2+1 / 4, \gamma / 2+1 / 2)$ and $E_{2}(\gamma)=(\gamma / 4+$ $1 / 8, \gamma / 4+1 / 4)$ are intervals, and $E_{1}(\gamma)$ is to the right of $E_{2}(\gamma)$. Now $\left|E_{1}(\gamma)\right|_{\gamma}=1 / 4$, and $\left|E_{2}(\gamma)\right|_{\gamma}=2\left|E_{2}(\gamma)\right|=1 / 4$, and the interval $[\gamma / 4+1 / 4, \gamma / 2+1 / 4]$ between $E_{2}$ and $E_{1}$ has strictly positive $e_{\gamma}$-mass, since $e_{\gamma} \geq 1$ on all of $[\gamma, \gamma+1 / 2]$. Consequently, both the interval consisting of those points of $[\gamma, \gamma+1 / 2]$ weakly to the left of $E_{1}(\gamma)$, and the interval consisting of those points of $[\gamma, \gamma+1 / 2]$ weakly to the right of $E_{2}(\gamma)$, have $e_{\gamma}$-mass strictly greater than $1 / 2$. The result then follows from Lemma 6.2.

For $^{9} \gamma \in \varrho^{-1}(0,1 / 2]$, the following surprising result, first discovered experimentally, asserts that $\omega(\gamma)$ first enters the flat spot $F_{\gamma}=(\gamma+1 / 2, \gamma)$ after either $1,2,4,5$ or 12 iterates, provided $\varrho(\gamma)$ is not equal to either $0,2 / 7,3 / 10,1 / 3$, or $3 / 8$.

Theorem 6.4. Provided $\varrho(\gamma) \notin\{0, \pm 2 / 7, \pm 3 / 10, \pm 1 / 3, \pm 3 / 8\}$, the entrance time $e_{\gamma}(\omega(\gamma))$ can only take one of the five values 1, 2, 4, 5, or 12. More precisely, for $\gamma \in \varrho^{-1}(0,1 / 2]$,

$$
\omega(\gamma) \in \begin{cases}E_{2}(\gamma) & \text { if } \varrho(\gamma) \in(0,2 / 7) \\ E_{12}(\gamma) & \text { if } \varrho(\gamma) \in(2 / 7,3 / 10) \\ E_{5}(\gamma) & \text { if } \varrho(\gamma) \in(3 / 10,1 / 3) \\ E_{4}(\gamma) & \text { if } \varrho(\gamma) \in(1 / 3,3 / 8) \\ E_{1}(\gamma) & \text { if } \varrho(\gamma) \in(3 / 8,1 / 2] .\end{cases}
$$

Proof. The case $\gamma \in \varrho^{-1}(0,2 / 7)$ is conveniently handled by first considering two sub-cases: $\gamma \in\left(\gamma_{\max }(0), \gamma_{\min }(1 / 4)\right]$ and $\gamma \in\left[\gamma_{\max }(1 / 4), \gamma_{\min }(2 / 7)\right)$. In the first sub-case ${ }^{10}$,

$$
E_{4}(\gamma) \prec E_{3}(\gamma) \prec E_{2}(\gamma)
$$

\footnotetext{
${ }^{9}$ By symmetry, the same is true for $\gamma \in \varrho^{-1}(-1 / 2,0]$, provided $\varrho(\gamma) \neq 0,-2 / 7,-3 / 10,-1 / 3,-3 / 8$.

${ }^{10}$ The ordering (16), in common with the other relative orderings (17), (18), (19), (20), can be proved by explicit calculation, the details of which will be omitted. An alternative justification follows from the fact that if $p / q<p^{\prime} / q^{\prime}$ are neighbouring rationals on some level of the Farey tree (see e.g. [13, Ch. III]), then for all $\varrho \in\left(p / q, p^{\prime} / q^{\prime}\right)$, the relative ordering of the points $a_{n}:=-n \varrho(\bmod 1), 1 \leq n \leq q+q^{\prime}-1$, is
} 
and $\left|E_{4}(\gamma) \cup E_{3}(\gamma) \cup E_{2}(\gamma)\right|_{\gamma}=4 / 2^{5}+3 / 2^{4}+2 / 2^{3}=9 / 16>1 / 2$, so $\omega(\gamma)$ is weakly to the left of $E_{2}(\gamma)$. But by Lemma $6.3, \omega(\gamma)$ is weakly to the right of $E_{2}(\gamma)$, so in fact $\omega(\gamma) \in E_{2}(\gamma)$. In the second sub-case,

$$
E_{7}(\gamma) \prec E_{3}(\gamma) \prec E_{6}(\gamma) \prec E_{2}(\gamma),
$$

and $\left|E_{7}(\gamma) \cup E_{3}(\gamma) \cup E_{6}(\gamma) \cup E_{2}(\gamma)\right|_{\gamma}=7 / 2^{8}+3 / 2^{4}+6 / 2^{7}+2 / 2^{3}=131 / 256>1 / 2$, so $\omega(\gamma)$ is weakly to the left of $E_{2}(\gamma)$, hence again $\omega(\gamma) \in E_{2}(\gamma)$ by Lemma 6.3. The fact that $\omega(\gamma) \in E_{2}(\gamma)$ in the remaining sub-case $\gamma \in\left[\gamma_{\min }(1 / 4), \gamma_{\max }(1 / 4)\right]$ follows, by Lemma 6.1, from the fact that it is true when $\gamma$ is either of the endpoints $\gamma_{\min }(1 / 4)$ or $\gamma_{\max }(1 / 4)$.

If $\gamma \in \varrho^{-1}(2 / 7,3 / 10)$ then necessarily ${ }^{11} q(\gamma) \geq 17$, so $E_{n}=E_{n}(\gamma)$ is an interval for $1 \leq n \leq 16$ by Theorem 3.13 , and

$$
E_{10} \prec E_{3} \prec E_{13} \prec E_{6} \prec E_{9} \prec E_{2} \prec E_{12} \prec E_{5} \prec E_{8} \prec E_{1} \prec E_{11} \prec E_{4} \prec E_{7} \text {. }
$$

Now $\left|E_{10} \cup E_{3} \cup E_{13} \cup E_{6} \cup E_{9} \cup E_{2} \cup E_{12}\right|_{\gamma}=10 / 2^{11}+3 / 2^{4}+13 / 2^{14}+6 / 2^{7}+9 / 2^{10}+$ $2 / 2^{3}+12 / 2^{13}=\frac{8197}{16384}>\frac{1}{2}$, so $\omega(\gamma)$ is weakly to the left of $E_{12}$. On the other hand, $\left|E_{12} \cup E_{5} \cup E_{8} \cup E_{1} \cup E_{11} \cup E_{4} \cup E_{7}\right|_{\gamma}=12 / 2^{13}+5 / 2^{6}+8 / 2^{9}+1 / 2^{2}+11 / 2^{12}=\frac{2049}{4096}>\frac{1}{2}$, so $\omega(\gamma)$ is weakly to the right of $E_{12}$, and hence $\omega(\gamma) \in E_{12}=E_{12}(\gamma)$.

If $\varrho(\gamma) \in(3 / 10,1 / 3)$ then $q(\gamma) \geq 13$, so $E_{n}=E_{n}(\gamma)$ is an interval for $1 \leq n \leq 12$, and

$$
E_{3} \prec E_{6} \prec E_{9} \prec E_{2} \prec E_{5} \prec E_{8} \prec E_{1} \prec E_{4} \prec E_{7} \prec E_{10} \text {. }
$$

Now $\left|E_{3} \cup E_{6} \cup E_{9} \cup E_{2} \cup E_{5}\right|_{\gamma}=3 / 2^{4}+6 / 2^{7}+9 / 2^{10}+2 / 2^{3}+5 / 2^{6}=\frac{585}{1024}>\frac{1}{2}$, so $\omega(\gamma)$ is weakly to the left of $E_{5}$, and $\left|E_{5} \cup E_{8} \cup E_{1} \cup E_{4} \cup E_{7} \cup E_{10}\right|_{\gamma}=5 / 2^{6}+8 / 2^{9}+1 / 2^{2}+4 / 2^{5}+7 / 2^{8}=$ $\frac{513}{1024}>\frac{1}{2}$, so $\omega(\gamma)$ is weakly to the right of $E_{5}$, and hence $\omega(\gamma) \in E_{5}(\gamma)$.

If $\varrho(\gamma) \in(1 / 3,3 / 8)$ then $q(\gamma) \geq 11$, so $E_{n}=E_{n}(\gamma)$ is an interval for $1 \leq n \leq 10$, and

$$
E_{8} \prec E_{5} \prec E_{2} \prec E_{10} \prec E_{7} \prec E_{4} \prec E_{1} \prec E_{9} \prec E_{6} \prec E_{3} .
$$

Now $\left|E_{8} \cup E_{5} \cup E_{2} \cup E_{10} \cup E_{7} \cup E_{4}\right|_{\gamma}=8 / 2^{9}+5 / 2^{6}+2 / 2^{3}+10 / 2^{11}+7 / 2^{8}+4 / 2^{5}=\frac{513}{1024}>\frac{1}{2}$, so $\omega(\gamma)$ is weakly to the left of $E_{4}$, and $\left|E_{4} \cup E_{1} \cup E_{9} \cup E_{6} \cup E_{3}\right|_{\gamma}=4 / 2^{5}+1 / 2^{2}+9 / 2^{10}+$ $6 / 2^{7}+3 / 2^{4}=\frac{623}{1024}>\frac{1}{2}$, so $\omega(\gamma)$ is weakly to the right of $E_{4}$, and hence $\omega(\gamma) \in E_{4}(\gamma)$.

Lastly, the case $\varrho(\gamma) \in(3 / 8,1 / 2]$ is handled by splitting into four sub-cases, according to whether $\gamma$ lies in $\left(\gamma_{\max }(3 / 8), \gamma_{\min }(2 / 5)\right], \varrho^{-1}(2 / 5),\left[\gamma_{\max }(2 / 5), \gamma_{\min }(1 / 2)\right]$, or $\varrho^{-1}(1 / 2)$. In the first sub-case $\gamma \in\left(\gamma_{\max }(3 / 8), \gamma_{\min }(2 / 5)\right], E_{1} \prec E_{6} \prec E_{11} \prec E_{3} \prec E_{8}$, and $\mid E_{1} \cup$ $\left.E_{6} \cup E_{11} \cup E_{3} \cup E_{8}\right|_{\gamma}=1 / 2^{2}+6 / 2^{7}+11 / 2^{12}+3 / 2^{4}+8 / 2^{9}=\frac{1}{2}+\frac{11}{2^{12}}>\frac{1}{2}$, so Lemma 6.2 implies that $\omega(\gamma)$ is weakly to the right of $E_{1}$. But $\omega(\gamma)$ is weakly to the left of $E_{1}$ by Lemma 6.3 , so in fact $\omega(\gamma) \in E_{1}(\gamma)$. In the third sub-case, $\gamma \in\left[\gamma_{\max }(2 / 5), \gamma_{\min }(1 / 2)\right]$, $E_{1}(\gamma) \prec E_{3}(\gamma) \prec E_{5}(\gamma)$, and $\left|E_{1}(\gamma) \cup E_{3}(\gamma) \cup E_{5}(\gamma)\right|_{\gamma}=1 / 2^{2}+3 / 2^{4}+5 / 2^{6}=\frac{33}{64}>\frac{1}{2}$, so $\omega(\gamma)$ is weakly to the right of $E_{1}$. But $\omega(\gamma)$ is weakly to the left of $E_{1}$ by Lemma 6.3 , so in fact $\omega(\gamma) \in E_{1}(\gamma)$. The second sub-case, $\varrho(\gamma)=2 / 5$, now follows from the first and third sub-cases, by virtue of Lemma 6.1. Similarly, Lemma 6.1 can be used to handle the fourth sub-case, $\gamma \in \varrho^{-1}(1 / 2)$ : the third sub-case implies that $\omega\left(\gamma_{\min }(1 / 2)\right) \in E_{1}\left(\gamma_{\min }(1 / 2)\right)$, and an analogous calculation shows that $\omega\left(\gamma_{\max }(1 / 2)\right) \in E_{1}\left(\gamma_{\max }(1 / 2)\right)$, so Lemma 6.1 implies that $\omega(\gamma) \in E_{1}(\gamma)$ for all $\gamma \in\left[\gamma_{\min }(1 / 2), \gamma_{\max }(1 / 2)\right]=\varrho^{-1}(1 / 2)$.

identical, and this ordering coincides with that of the $E_{n}(\gamma), 1 \leq n \leq q+q^{\prime}-1$, in the sense that $a_{m}<a_{n}$ if and only if $E_{m}(\gamma) \prec E_{n}(\gamma)$.

${ }^{11}$ We use $q(\gamma)$ to denote the denominator of the rational $\varrho(\gamma)=p(\gamma) / q(\gamma)$ when expressed in lowest terms; if $\varrho(\gamma) \notin \mathbb{Q}$ we define $q(\gamma)=\infty$. 
Remark 6.5. The proof of Theorem 6.4 in fact shows that $\omega(\gamma) \in E_{2}(\gamma)$ for all $\gamma \in$ $\left(\gamma_{\max }(0), \gamma_{\min }(2 / 7)\right]$ (note that $\left.\omega\left(\gamma_{\max }(0)\right)=1 / 8 \notin(1 / 8,1 / 4)=E_{2}\left(\gamma_{\max }(0)\right)\right)$. Similarly, $\omega(\gamma) \in E_{12}(\gamma)$ for $\gamma \in\left[\gamma_{\max }(2 / 7), \gamma_{\min }(3 / 10)\right], \omega(\gamma) \in E_{5}(\gamma)$ for $\gamma \in\left[\gamma_{\max }(3 / 10), \gamma_{\min }(1 / 3)\right]$, $\omega(\gamma) \in E_{4}(\gamma)$ for $\gamma \in\left[\gamma_{\max }(1 / 3), \gamma_{\min }(3 / 8)\right]$, and $\omega(\gamma) \in E_{1}(\gamma)$ for $\gamma \in\left[\gamma_{\max }(3 / 8), \gamma_{\min }(-3 / 8)\right]$.

Corollary 6.6. If $T_{\gamma}$ has rational rotation number $\varrho(\gamma)=p / q$ (where $q \in \mathbb{N}$ and the integer $0<p<q$ is coprime to $q$ ), then

$$
\omega(\gamma) \in \begin{cases}J_{q-2 p} & \text { if } \varrho(\gamma) \in(0,2 / 7) \\ J_{4 q-12 p} & \text { if } \varrho(\gamma) \in(2 / 7,3 / 10) \\ J_{2 q-5 p} & \text { if } \varrho(\gamma) \in(3 / 10,1 / 3) \\ J_{2 q-4 p} & \text { if } \varrho(\gamma) \in(1 / 3,3 / 8) \\ J_{q-p} & \text { if } \varrho(\gamma) \in(3 / 8,1 / 2] .\end{cases}
$$

Proof. If $\varrho(\gamma) \in(0,2 / 7)$ then, by Theorem 6.4, $\omega(\gamma) \in E_{2}(\gamma) \subset K_{2}=J_{\pi_{p, q}(2)}$. But $\pi_{p, q}(2)=-2 p(\bmod q)=q-2 p$ for $p / q \in(0,2 / 7)$. The other four cases are proved similarly.

\section{REFERENCES}

[1] H. van den Bedem and N. Chernov, Expanding maps of an interval with holes, Ergod. Th. \& Dyn. Sys., 22 (2002), 637-654.

[2] C. Boyd, On the structure of the family of Cherry fields on the torus, Ergod. Th. $\&$ Dyn. Sys., 5 (1985), 27-46.

[3] P. L. Boyland, Bifurcations of circle maps: Arnol'd tongues, bistability and rotation intervals, Comm. Math. Phys., 106 (1986), 353-381.

[4] S. Bullett \& P. Sentenac, Ordered orbits of the shift, square roots, and the devil's staircase, Math. Proc. Camb. Phil. Soc., 115 (1994), 451-481.

[5] L. A. Bunimovich \& A. Yurchenko, Where to place a hole to achieve a maximum escape rate, preprint, 2008.

[6] W. de Melo \& J. Palis, Geometric theory of dynamical systems. An introduction, Springer-Verlag, New York-Berlin, 1982.

[7] M. Demers, Markov extensions for dynamical systems with holes: an application to expanding maps of the interval, Israel J. Math., 146 (2005), 189-221.

[8] M. Demers \& L.-S. Young, Escape rates and conditionally invariant measures, Nonlinearity, 19 (2006), 377-397.

[9] J.-P. Eckmann \& D. Ruelle, Ergodic theory of chaos and strange attractors, Rev. Modern Phys., 57 (1985), 617-656.

[10] J.-M. Gambaudo, O. Lanford III, \& C. Tresser, Dynamique symbolique des rotations, C. R. Acad. Sc. Paris, 299 (1984), 823-826.

[11] M. Herman, Sur la conjugaison différentiable des difféomorphismes du cercle à des rotations, Publ. Math. IHES, 49 (1979), 5-233.

[12] J. Graczyk, L. B. Jonker, G. Swiatek, F. M. Tangerman, J. J. P. Veerman, Differentiable circle maps with a flat interval, Comm. Math. Phys., 173 (1995), 599-622.

[13] G. H. Hardy \& E. M. Wright, An introduction to the theory of numbers, 5th edition, Oxford University Press, 1979.

[14] G. Keller, Markov extensions, zeta functions, and Fredholm theory for piecewise invertible dynamical systems, Trans. Amer. Math. Soc., 314 (1989), 433-497.

[15] S. Newhouse, J. Palis and F. Takens, Bifurcations and stability of families of diffeomorphisms, Publ. Math. IHES, 57 (1983), 5-72.

[16] Z. Nitecki, Differentiable dynamics, MIT Press, Cambridge, Mass., 1971. 
[17] G. Pianigiani \& J. Yorke, Expanding maps on sets which are almost invariant: decay and chaos, Trans. Amer. Math. Soc., 252 (1979), 351-366.

[18] J. J. P. Veerman, Symbolic dynamics and rotation numbers, Physica A, 134 (1986), 543-576.

[19] J. J. P. Veerman, Symbolic dynamics of order-preserving orbits, Physica D, 29 (1987), 191-201.

[20] J. J. P. Veerman, Irrational rotation numbers, Nonlinearity, 2 (1989), 419-428.

[21] J. J. P. Veerman, Hausdorff dimension of order preserving sets, Comm. Math. Phys., 127 (1990), 313-317.

[22] L.-S. Young, What are SRB measures, and which dynamical systems have them?, J. Stat. Phys., 108 (2002), 733-754.

Vasso Anagnostopoulou; School of Mathematical Sciences, Queen Mary, University of London, Mile End RoAd, London, E1 4NS, UK.

vaa@maths.qmul.ac.uk

Karla Díaz-Ordaz; School of Mathematical Sciences, Queen Mary, University of LonDon, Mile End RoAd, London, E1 4NS, UK.

karla@maths.qmul.ac.uk

Oliver Jenkinson; School of Mathematical Sciences, Queen Mary, University of LonDon, Mile End RoAd, London, E1 4NS, UK.

omj@maths.qmul.ac.uk

Catherine Richard; School of Mathematical Sciences, Queen Mary, University of London, Mile End RoAd, London, E1 4NS, UK.

csr@maths . qmul . ac . uk 